\title{
Mode of action of the 2-phenylquinoline efflux inhibitor PQQ4R against Escherichia coli
}

\author{
Diana Machado $^{1}$, Laura Fernandes $^{1}$, Sofia S Costa ${ }^{1}$, Rolando Cannalire $^{2}{ }^{\text {, Giuseppe Manfroni }}{ }^{2}$, Oriana \\ Tabarrini $^{2}{ }^{\text {, Isabel Couto }}{ }^{1}$, Stefano Sabatini ${ }^{2}$, Miguel Viveiros ${ }^{\text {Corresp. }}{ }^{1}$ \\ ${ }^{1}$ Unidade de Microbiologia Médica, Global Health and Tropical Medicine, GHTM, Instituto de Higiene e Medicina Tropical, IHMT, Universidade NOVA de \\ Lisboa, UNL, Lisboa, Portugal \\ 2 Department of Pharmaceutical Sciences, Università degli Studi di Perugia, Perugia, Italy \\ Corresponding Author: Miguel Viveiros \\ Email address: mviveiros@ihmt.unl.pt
}

Efflux pump inhibitors are of great interest since their use as adjuvants of bacterial chemotherapy can increase the intracellular concentrations of the antibiotics and assist in the battle against the rising of antibiotic-resistant bacteria. In this work, we have described the mode of action of the 2-phenylquinoline efflux inhibitor (4-(2-(piperazin-1-yl)ethoxy)-2(4-propoxyphenyl) quinolone - PQQ4R), against Escherichia coli, by studding its efflux inhibitory ability, its synergistic activity in combination with antibiotics, and compared its effects with the inhibitors phenyl-arginine- $\beta$-naphthylamide (PABN) and chlorpromazine (CPZ). The results showed that PQQ4R acts synergistically, in a concentration dependent manner, with antibiotics known to be subject to efflux in $E$. coli reducing their MIC in correlation with the inhibition of their efflux. Real-time fluorometry assays demonstrated that PQQ4R at sub-inhibitory concentrations promote the intracellular accumulation of ethidium bromide inhibiting its efflux similarly to $P A B N$ or $C P Z$, well-known and described efflux pump inhibitors for Gram-negative bacteria and whose clinical usage is limited by their levels of toxicity at clinical and bacteriological effective concentrations. The time-kill studies showed that PQQ4R, at bactericidal concentrations, has a rapid antimicrobial activity associated with a fast decrease of the intracellular ATP levels. The results also indicated that the mode of action of PQQ4R involves the destabilization of the $E$. coli inner membrane potential and ATP production impairment, ultimately leading to efflux pump inhibition by interference with the energy required by the efflux systems. At bactericidal concentrations, membrane permeabilization increases and finally ATP is totally depleted leading to cell death. Since drug resistance mediated by the activity of efflux pumps depends largely on the proton motive force (PMF), dissipaters of PMF such as PQQ4R, can be regarded as future adjuvants of conventional therapy against $E$. coli and other Gramnegative bacteria, especially their multidrug resistant forms. Their major limitation is the high toxicity for human cells at the concentrations needed to be effective against bacteria. 
Their future molecular optimization to improve the efflux inhibitory properties and reduce relative toxicity will optimize their potential for clinical usage against multi-drug resistant bacterial infections due to efflux. 


\section{Mode of action of the 2 -phenylquinoline efflux inhibitor \\ 2 PQQ4R against Escherichia coli}

3 Diana Machado ${ }^{1}$, Laura Fernandes ${ }^{1, \&}$, Sofia Santos Costa ${ }^{~}$, Rolando Cannalire ${ }^{2}$, Giuseppe Manfroni ${ }^{2}$,

4 Oriana Tabarrini ${ }^{2}$, Isabel Couto ${ }^{1}$, Stefano Sabatini ${ }^{2}$ and Miguel Viveiros ${ }^{1, *}$

$5{ }^{1}$ Unidade de Microbiologia Médica, Global Health and Tropical Medicine, GHTM, Instituto de Higiene e

6 Medicina Tropical, IHMT, Universidade NOVA de Lisboa, UNL, Lisboa, Portugal

$7 \quad{ }^{2}$ Department of Pharmaceutical Sciences, Università degli Studi di Perugia, Perugia, Italy

8 \& Present address: Laboratório de Diagnóstico Molecular Veterinário GeneVet, Algés, Portugal

Corresponding Author:

11 Miguel Viveiros ${ }^{1, *}$

12 Rua da Junqueira 100, Lisboa, 1349-008, Portugal

Email address: mviveiros@ihmt.unl.pt 


\section{ABSTRACT}

Efflux pump inhibitors are of great interest since their use as adjuvants of bacterial chemotherapy can increase the intracellular concentrations of the antibiotics and assist in the battle against the rising of antibiotic-resistant bacteria. In this work, we have described the mode of action of the 2-phenylquinoline efflux inhibitor (4-(2-(piperazin-1-yl)ethoxy)-2-(4propoxyphenyl) quinolone - PQQ4R), against Escherichia coli, by studding its efflux inhibitory ability, its synergistic activity in combination with antibiotics, and compared its effects with the inhibitors phenyl-arginine- $\beta$-naphthylamide (PA $\beta N)$ and chlorpromazine (CPZ).

The results showed that PQQ4R acts synergistically, in a concentration dependent manner, with antibiotics known to be subject to efflux in E. coli reducing their MIC in correlation with the inhibition of their efflux. Real-time fluorometry assays demonstrated that PQQ4R at subinhibitory concentrations promote the intracellular accumulation of ethidium bromide inhibiting its efflux similarly to $\mathrm{PA} \beta \mathrm{N}$ or $\mathrm{CPZ}$, well-known and described efflux pump inhibitors for Gram-negative bacteria and whose clinical usage is limited by their levels of toxicity at clinical and bacteriological effective concentrations. The time-kill studies showed that PQQ4R, at bactericidal concentrations, has a rapid antimicrobial activity associated with a fast decrease of the intracellular ATP levels. The results also indicated that the mode of action of PQQ4R involves the destabilization of the $E$. coli inner membrane potential and ATP production impairment, ultimately leading to efflux pump inhibition by interference with the energy required by the efflux systems. At bactericidal concentrations, membrane permeabilization increases and finally ATP is totally depleted leading to cell death. Since drug resistance mediated by the activity of efflux pumps depends largely on the proton motive force (PMF), dissipaters of PMF such as PQQ4R, can be regarded as future adjuvants of conventional therapy against $E$. coli and other Gram-negative bacteria, especially their multidrug resistant forms. Their major limitation is the high toxicity for human cells at the concentrations needed to be effective against bacteria. Their future molecular optimization to improve the efflux inhibitory properties and reduce relative toxicity will optimize their potential for clinical usage against multi-drug resistant bacterial infections due to efflux. 


\section{INTRODUCTION}

The emergence of drug resistant bacteria represent a global threat to human health and is now a top priority of the World Health Organization (WHO) and the European Centre for Disease Prevention and Control (ECDC) programs for the treatment of infectious diseases. Particularly, the emergence of drug resistance in Escherichia coli requires close attention since the rate of isolates resistant to the commonly used antibiotics is rising worldwide (World Health Organization, 2014; European Centre for Disease Prevention and Control, 2015). The current therapeutic options are scarce to deal with these infections. Therefore, studies on new drugs and drug combinations as well as an improved understanding of the mechanism of action of these new drugs have become critical to fight the spread of multidrug resistant organisms. In Gram-negative bacteria, besides the acquired resistance by the acquisition of external resistance determinants or mutations in genes that code for the drug targets, the intrinsic drug resistance also play an important role in the resistance towards antibiotics and biocides (Viveiros et al., 2007; Piddock, 2006; Piddock, 2007; Nikaido \& Pagès, 2012). This resistance occurs as consequence of the (i) presence of an outer membrane that create a permeability barrier reducing the influx of antimicrobials, and (ii) overexpression of efflux pumps that help to reduce the intracellular level of antimicrobials and toxins (Nikaido \& Pagès, 2012; Piddock, 2006).

The efflux pumps of the RND (resistant nodulation cell division) superfamily have been clearly associated with multidrug resistant phenotypes in Gram-negative pathogens (Nikaido \& Pagès, 2012). The substrates of the RND efflux pumps are different in their structure and physicochemical properties and include antibiotics, detergents, and biocides (Piddock, 2006; Li \& Nikaido, 2009). The clinical implication of this substrate promiscuity is the development of multidrug resistance. The major RND efflux system of $E$. coli consists in a typical tripartite efflux pump, the AcrAB-TolC. This structure is composed by an integral membrane efflux transporter (AcrB), an outer membrane channel (TolC), and a periplasmic adapter protein (AcrA) (Du et al., 2014). Upon entering in the cell, the compounds will interact with the substrate-binding pocket of AcrB, which will extrude the compounds via TolC using the energy produced by the proton motive force (PMF) (Nikaido \& Takatsuka, 2009). The AcrAB activity and overexpression have 
84

85

86

87

been associated with the resistance to fluoroquinolones, chloramphenicol, tetracycline, $\beta$ lactams, and $\beta$-lactamase inhibitors, among others, as well as biofilm formation and pathogenicity (Piddock, 2007). Beside the AcrAB-TolC efflux pump, other $E$. coli efflux systems also play a role in the development of drug resistance (Viveiros et al., 2007; Nishino et al., 2003). The overexpression of these efflux systems in response to the antibiotic stress is commonly the first step in the evolution of antibiotic resistance in the bacterial population, favoring the spontaneous appearance and stabilization of chromosomal mutations in the genes related with the antibiotic action. This biological phenomenon leads to the emergence of resistance to almost all classes of antibiotics available and require the urgent implementation of novel therapeutic strategies for the management of these infections.

The inhibition of the efflux activity may be of great potential once used in combination therapies by restoring or enhancing the activity of the currently used antimicrobials and preventing the emergence of drug resistance. Several compounds capable of inhibiting $E$. coli efflux pumps have been described (Lomovskaya et al., 2001; Viveiros et al., 2005; Bohnert, Schuster \& Kern, 2013; Opperman et al., 2014; Vargiu et al., 2014; Yilmaz et al., 2015; Bohnert et al., 2016; Zuo, Weng \& Wang, 2016) but, to date, none has reached the clinical development mainly due to their toxicity. Moreover, the putative mechanisms of action of the great majority of the efflux inhibitors currently in study remain largely unknown. The most known $E$. coli efflux inhibitors are the peptidomimetic phenyl-arginine- $\beta$-naphthylamide (PABN) and the phenothiazine chlorpromazine (CPZ). PA $3 N$ is a potent inhibitor of Pseudomonas aeruginosa efflux pumps (Lomovskaya et al., 2001) and is a substrate competitor of the AcrAB efflux pump of E. coli (Viveiros et al., 2008). Recently, Misra et al. (2015) showed that PA $\beta N$ acts as an inhibitor of the AcrAB and AcrEF efflux systems at low concentrations, and as a membranedestabilizing agent when used at higher concentrations. The phenothiazine CPZ presents efflux inhibitory activity against $E$. coli efflux systems (Viveiros et al, 2005). The phenothiazines mode of action involve the disruption of the calcium-calmodulin transport and signaling pathways (Salih et al, 1991; Pluta et al, 2011), modifications at the level of the bacterial membrane and on nucleic acid stability (Pluta et al, 2011; Thorsing et al, 2013), and inhibition of the type II NADH-ubiquinone dehydrogenase (Weinstein et al, 2005; Schurig-Briccio et al., 2014). 
113 In recent studies, the 2-phenylquinoline derivative PQQ4R showed to be active as an efflux

114 pump inhibitor of the Gram-positive S. aureus (Sabatini et al., 2011; Sabatini et al., 2013) and

115 non-tuberculous mycobacteria (Machado et al., 2015) but nothing is known about its mode of

116 action. In this work, we aimed to unravel the mode of action of PQQ4R as efflux pump inhibitor,

117 as in the search for new and effective efflux inhibitors is important to understand their

118 inhibitory mechanism of action and to disclosure the presence of non-efflux related

119 mechanisms (Venter et al., 2015). Because drug efflux is energy dependent, we hypothesize

120 that the mode by which PQQ4R inhibits drug efflux involves the interference with the cell

121 energetic state. We evaluated the activity of PQQ4R using three $E$. coli isogenic strains differing

122 only in the level of expression of its major efflux pump system - the AcrAB-TolC system - and

123 compared its activity with that of the known E. coli efflux inhibitors PABN and CPZ. We found

124 that PQQ4R interferes with E. coli membrane integrity, therefore reducing the activity of $E$. coli

125 efflux pumps. The understanding gathered on the molecular mechanism of action of the efflux

126 inhibitor PQQ4R may aid in the development of less toxic and more potent efflux inhibitors

127 against $E$. coli efflux pumps.

128

129 MATERIALS \& METHODS

130 Bacteria and growth conditions

131 The strains included in the study were the wild-type E. coli K-12 AG100 (argE3 thi-1 rpsL xyl mtl

$132 \Delta$ (gal-uvrB)supE44); the AcrAB pump-deficient E. coli AG100A ( $\triangle \mathrm{acrAB})$, and the AcrAB

133 overexpressing E. coli $A G 100_{\text {tet }}$. The strain AG100A is a derivative of AG100 and has the AcrAB

134 system inactivated due to an insertion of the transposon Tn903 ( $\triangle$ acrAB::Tn903 Kan') (George \&

135 Levy, 1983; Okusu \& Nikaido, 1996). AG100 ${ }_{\text {tet }}$ is a derivative of AG100 obtained by continuous

136 exposure to increasing concentrations of tetracycline (TET) (Viveiros et al., 2005). The strains

137 were grown in Luria-Bertani (LB) broth at $37^{\circ} \mathrm{C}$ with shaking. AG100A was grown in presence

138 kanamycin (KAN) at $100 \mu \mathrm{g} / \mathrm{ml}$ to maintain the transposon and $A G 100_{\text {tet }}$ was grown in media

139 supplemented with TET at $8 \mu \mathrm{g} / \mathrm{ml}$ to maintain the overexpression of efflux pumps.

140

141

Chemicals 
142 Ofloxacin (OFX), oxacillin (OXA), KAN, TET, ethidium bromide (EtBr), CPZ, PAßN, carbonyl 143 cyanide-m-chlorophenylhydrazone (CCCP), glucose, and phosphate-buffered saline (PBS) were 144 purchased from Sigma-Aldrich (St. Louis, MO, USA). The 2-phenylquinoline PQQ4R was 145 synthesized as previously described (Sabatini et al., 2013).

\section{Antibacterial activity evaluation}

The minimum inhibitory concentrations (MICs) were determined using the broth microdilution method according to the CLSI guidelines (Clinical and Laboratory Standards Institute, 2014). The synergistic activity between the efflux inhibitors, antibiotics and $\mathrm{EtBr}$ was evaluated by checkerboard assays as previously described (Pillai, Moellering \& Eliopoulos, 2005; Coelho et al., 2015). Two-fold serial dilutions of the compounds were made to achieve the following concentrations: $320 \mu \mathrm{M}$ to $40 \mu \mathrm{M}$. The MICs were determined as the lowest concentration at which no visible growth was observed after 18 hours of incubation at $37^{\circ} \mathrm{C}$. The assays were performed in triplicate and the MIC value was given as result of at least two concordant values.

\section{Evaluation of efflux activity by real-time fluorometry}

158

159

160

161

162

163

164

165

166

167

168

169

170

The effect of the inhibitors on $\mathrm{EtBr}$ accumulation and efflux was assessed by fluorometry as previously described (Viveiros et al., 2008; Viveiros et al., 2010). The strains were grown until an $\mathrm{OD}_{600 \mathrm{~nm}}$ of 0.6 at $37^{\circ} \mathrm{C}$ with shaking. After, the cells were collected by centrifugation at $16060 \mathrm{x}$ $\mathrm{g}$ for 3 minutes, washed in PBS and centrifuged again.

For the accumulation assays, the $\mathrm{OD}_{600 \mathrm{~nm}}$ of the cell suspension was adjusted to 0.6 by adding PBS, allowing the assay to run with a final OD of 0.3 . To determine the EtBr concentration at which influx and efflux are in equilibrium, the accumulation assays were performed in presence of increasing concentrations of the dye. The assays were prepared to a final volume of $100 \mu l$ containing $50 \mu \mathrm{l}$ of the cellular suspension (final $\mathrm{OD}_{600 \mathrm{~nm}}$ of 0.3 ) plus $50 \mu \mathrm{l}$ of EtBr solutions to final concentrations ranging from 0.0625 to $5 \mu \mathrm{g} / \mathrm{ml}$. The effect of the inhibitors on the accumulation of EtBr was evaluated in a final volume of $100 \mu$ l containing $50 \mu$ of the cellular suspension (final $\mathrm{OD}_{600 \mathrm{~nm}}$ of 0.3 ) and $50 \mu \mathrm{l}$ of a solution containing $\mathrm{EtBr}$ at the equilibrium concentration and the compounds to a final concentration of $80 \mu \mathrm{M}$ for $A G 100$ and $A G 100_{\text {tet }}$ 
171 and $20 \mu \mathrm{M}$ for AG100A. The molar concentrations used represent $1 / 4$ or less of the MIC

172 determined for each compound against each strain (see Table 1) in order to guarantee that the

173 real-time efflux inhibitory effects measured were not due to any antimicrobial effect of the

174 compound. The assays were conducted in a Rotor-Gene 3000 (Corbett Research, Sydney,

175 Australia) at $37^{\circ} \mathrm{C}$, and the fluorescence acquired at $530 / 585 \mathrm{~nm}$ at the end of every 60

176 seconds, for 30 minutes. The activity of the compounds on the accumulation of EtBr was

177 evaluated by the relative final fluorescence (RFF) index according to the formula: $R F F=\left(R_{\text {treated }}{ }^{-}\right.$

$\left.178 \mathrm{RF}_{\text {untreated }}\right) / \mathrm{RF}_{\text {untreated. }}$ In this formulae the $\mathrm{RF}_{\text {treated }}$ corresponds to the fluorescence at the last

179 time point of the EtBr accumulation curve (minute 30) in the presence of an inhibitor and the

$180 \mathrm{RF}_{\text {untreated }}$ corresponds to the fluorescence at the last time point of the EtBr accumulation curve

181 of the control tube (Machado et al., 2011). The experiments were done in triplicate and the RFF

182 values are presented as the average of three independent assays ( \pm SD).

183 For the efflux assays, the strains were exposed to conditions that promote maximum

184 accumulation of $\mathrm{EtBr}$, i.e., EtBr at the equilibrium concentration for each strain, no glucose,

185 presence of the efflux inhibitors, and incubation at room temperature for 1 hour (Viveiros et al.,

186 2010). The $\mathrm{OD}_{600 \mathrm{~nm}}$ of the cell suspension was adjusted to 0.3 and incubated with $\mathrm{EtBr}$ under the

187 conditions described above. Aliquots of $50 \mu \mathrm{l}$ of the cells were transferred to tubes containing

$18850 \mu \mathrm{l}$ of each efflux inhibitor at $80 \mu \mathrm{M}$ (AG100 and $A G 100_{\text {tet }}$ ) or $20 \mu \mathrm{M}$ (AG100A) without EtBr.

189 Control tubes with only cells and cells with and without $0.4 \%$ glucose were included. The

190 fluorescence was measured in a Rotor-Gene 3000 and the data was acquired every 30 seconds

191 for 30 minutes at $37^{\circ} \mathrm{C}$. The efflux activity was quantified by comparing the fluorescence data

192 obtained under conditions that promote efflux (presence of glucose and absence of efflux

193 inhibitor) with the data from the control in which the bacteria are under conditions of no efflux

194 (presence of an inhibitor and no energy source). The relative fluorescence corresponds to the

195 ratio of the fluorescence that remains per unit of time, relatively to the EtBr-loaded cells

196 (Viveiros et al., 2008; Viveiros et al., 2010).

197

198

Time-kill kinetics 
199 The determination of the killing activity of PQQ4R was analysed by time-kill assays as previously 200 described (Pillai, Moellering \& Eliopoulos, 2005) with slight modifications. Briefly, AG100 was 201 grown until $\mathrm{OD}_{600 \mathrm{~nm}}$ of 0.6 at $37^{\circ} \mathrm{C}$ with shaking. Exponential bacterial cultures were diluted in 202 Mueller-Hinton Broth (MHB) to a cell density of $1 \times 10^{5}$ cells $/ \mathrm{ml}$ and $500 \mu \mathrm{l}$ added to test tubes 203 containing MHB and PQQ4R at the desired concentration. The compound was added to each 204 tube to achieve the final concentrations of $8 x$ to $0.5 x$ the MIC. A drug-free control was included 205 in the assay to monitor the normal growth of the strain. Cultures were sampled for CFU 206 determination after $0,1,2,3,4,5,6$, and 24 hours of incubation at $37^{\circ} \mathrm{C}$ with shaking. For CFU 207 determination, 10 -fold serial dilutions were made in a saline solution and $20 \mu$ of each solution 208 was placed onto the surface of a Mueller-Hinton agar plate. The colonies were counted after the incubation of the plates at $37^{\circ} \mathrm{C}$ for 24 hours. The limit of detection of the assay was 17 $\mathrm{CFU} / \mathrm{ml}$. Each assay was repeated at least twice.

211

\section{Membrane potential assay}

213 The effect of PQQ4R on the membrane potential was measured using the BacLight Bacterial Membrane Potential Kit (Molecular Probes, Life Technologies) according to the manufacturer's instructions. $\mathrm{CPZ}$ and $\mathrm{PA} \beta \mathrm{N}$ were tested for comparison. $\mathrm{AG} 100$ was grown at $37^{\circ} \mathrm{C}$ with shaking until reach $\mathrm{OD}_{600 \mathrm{~nm}}$ 0.6. After this, the cells were washed in PBS and diluted to $1 \times 10^{7} \mathrm{CFU} / \mathrm{ml}$ with PBS. PQQ4R was added to the cell suspension at concentrations from 640 to $80 \mu \mathrm{M}$. The samples were transferred into black flat bottom 96-well plates, $30 \mu \mathrm{M}$ of $\mathrm{DiOC}_{2}(3)$ was added to the mixture, and the plates were incubated during 30 minutes in the dark. The fluorescence was measured using a Synergy HT multi-mode microplate reader (BioTek Instruments Inc, Vermont, USA) with the filters 485/20 (excitation) and 528/20 (emission) for green and 590/35 (emission) for red. CCCP was used as positive control at $156 \mu \mathrm{M}$ (half MIC), since it eradicates the proton gradient, eliminating the membrane potential. The red to green ratio was determined and normalized against the emission from the $\mathrm{DiOC}_{2}(3)$ blank well and the results are presented as the percentage of depolarized membranes $( \pm S D)$ compared with the drug-free control. 


\section{Membrane permeability assay}

229 The evaluation of the membrane integrity was done using the Live/Dead BacLight Bacterial 230 Viability Kit (Molecular Probes, Life Technologies) according to the manufacturer's instructions. 231 AG100 was grown at $37^{\circ} \mathrm{C}$ with shaking until $O_{600 \mathrm{~nm}}$ of 0.6 . Samples of $500 \mu$ l were incubated 232 with PQQ4R at concentrations from 640 to $80 \mu \mathrm{M}$ during 1 hour at room temperature. The cells 233 were collected by centrifugation at $16060 \mathrm{xg}$ for 10 minutes, the supernatant was discarded 234 and the pellet resuspended in the same volume of a saline solution. Then, $100 \mu$ l of the cell 235 suspension were transferred into black flat bottom 96-well plates and the dyes propidium 236 iodide and SYTO 9 (ratio 1:1) were added to each well to stain the cells. The plate was 237 incubated during 15 minutes at room temperature in the dark. The fluorescence was measured 238 using a Synergy HT multi-mode microplate reader with the filters 485/20 (excitation) and 239 528/20 (emission) for green and 590/35 (emission) for red. The green to red ratio was 240 determined and the results are presented as the percentage of intact membranes ( \pm SD) compared with the control (no treatment). CPZ and PAßN were included for comparison.

\section{Intracellular ATP levels determination}

The ATP levels were measured using the ATP Determination Kit (Invitrogen, Life Technologies, Paisley, UK) according to the manufacturer's instructions. AG100 was grown until $\mathrm{OD}_{600 \mathrm{~nm}}$ of 0.6 at $37^{\circ} \mathrm{C}$ with shaking. Exponential bacterial cultures were diluted in $\mathrm{MHB}$ to a cell density of $1 \times 10^{5} \mathrm{cells} / \mathrm{ml}$ and $500 \mu \mathrm{l}$ added to test tubes containing MHB and PQQ4R at half MIC and at the MIC. A drug-free control was included in the assay to monitor the normal growth of the strain. Aliquots of bacteria were collected at $0,1,2,3,4,5,6$, and 24 hours of incubation at $37^{\circ} \mathrm{C}$, with shaking, inactivated by heating and immediately deep frozen. The cell lysates were transferred into white flat bottom 96-well plates and the ATP content measured using a Synergy HT multi-mode microplate reader and expressed as relative luminescence units. CPZ and PABN were included for comparison.

\section{Cytotoxicity against human monocyte-derived macrophages}


256 The blood was collected from healthy donors and the peripheral blood mononuclear cells were

257 isolated by Ficoll-Paque Plus (GE Healthcare, Freiburg, Germany) density gradient centrifugation

258 as previously described (Machado et al., 2016). Briefly, monocytes were differentiated into

259 macrophages during 7 days in RPMI-1640 medium with $10 \%$ fetal calf serum (FCS), $1 \%$

260 GlutaMAX'⿳一, $1 \mathrm{mM}$ sodium pyruvate, $10 \mathrm{mM}$ HEPES at $\mathrm{pH}$ 7.4, $100 \mathrm{IU} / \mathrm{ml}$ penicillin and 100

$261 \mu \mathrm{g} / \mathrm{ml}$ streptomycin (Gibco, Life Technologies), and 20 ng/ml M-CSF (Immunotools, Friesoythe,

262 Germany) and incubated at $37^{\circ} \mathrm{C}$ in a $5 \% \mathrm{CO}_{2}$ atmosphere. Fresh medium was added at day 4

263 post isolation. The effect of the compounds on human monocyte-derived macrophages was

264 evaluated using the AlamarBlue method (Molecular Probes, Life Technologies) (O'Brien et al.,

265 2000), and by measuring the release of the lactate dehydrogenase (LDH) into the culture

266 supernatants using the Pierce LDH Cytotoxicity Assay (ThermoFisher Scientific, Waltham, MA,

267 USA) (Chan et al., 2013) according to the manufacturer's instructions. Briefly, 5x10 4 cells were

268 seeded in 96-well microplates treated with the compounds, and incubated at $37^{\circ} \mathrm{C}$ with $5 \% \mathrm{CO}_{2}$.

269 After 72 hours of treatment, the cell viability was assessed. For the AlamarBlue method, the

270 dye was added to each well to a final concentration of $10 \%$ and incubated overnight at $37^{\circ} \mathrm{C}$

271 and $5 \% \mathrm{CO}_{2}$. The fluorescence was measured with a 540/35 excitation filter and a 590/20

272 emission filter in a Synergy HT multi-mode microplate reader. The release of LDH from

273 damaged cells into culture supernatants, as an indicator of cytotoxicity, was measured in

274 serum-free medium. Here the amount of enzyme activity correlates to the number of damaged

275 cells. The specific lysis was calculated as follows: (treated cells - spontaneous LDH release)/

276 (maximum LDH release - spontaneous LDH release) x100. The inhibitory concentration IC ${ }_{50}$

277 (50\%) value was calculated using GraphPad Prism V5.01 software (La Jolla, USA). The IC 10 (10\%)

278 and IC 90 (90\%) were calculated using the GraphPad program "ECanything" available online

279 (GraphPad Software QuickCalcs, 2016).

280

281 Statistical analysis

282 Statistical analysis was carried out using the Student's t-test. A P value $<0.05$ was considered

283 statistically significant and highly significant when $* * P<0.01$ and $* * * P<0.001$ (two-tailed 284 tested). 


\section{RESULTS}

\section{Synergistic activity of PQQ4R in combination with antimicrobials}

288

289

290

291

292

293

294

295

296

297

298

299

300

301

302

303

304

305

306

307

308

309

310

311

312

313

The MICs of the antibiotics, which are substrates of the AcrAB efflux pump, the efflux substrate $\mathrm{EtBr}, \mathrm{PQQ} 4 \mathrm{R}$, and for comparison, $\mathrm{PA} \beta \mathrm{N}$ and $\mathrm{CPZ}$, known E. coli efflux inhibitors, were determined for the wild-type AG100, the pump deficient AG100A, and the pumpoverexpressing $A G 100_{\text {tet }}$ (Table 1). AG100A is more susceptible to the selected antibiotics and $\mathrm{EtBr}$ than the wild-type, due to the absence of a functional AcrAB, reconfirming that these antibiotics are substrates of this pump (Piddock, 2006; Viveiros et al., 2005). The susceptibility data also showed that neither PQQ4R nor PABN and CPZ had, by themselves, antimicrobial activity against the $E$. coli strains. Moreover, PQQ4R MIC decreased 8-fold against AG100A once compared with the wild-type parental strain, indicating that, PQQ4R might be a substrate of the AcrAB system. Similar results were observed for PABN.

To assess whether PQQ4R potentiated the activity of the tested antibiotics against $E$. coli, the MICs of OFX, OXA, and TET were determined in the presence of PQQ4R. PA $B N$ and CPZ were tested at the same concentrations for a direct comparison of their activities (Table 2). Against the wild-type strain, PQQ4R, at concentrations ranging from 40 to $160 \mu \mathrm{M}$, produced a 2-fold reduction in OFX and TET MICs, while a 2- or 4-fold reduction on the MICs of EtBr at 80 and 160 $\mu \mathrm{M}$, respectively, were observed. On the contrary, no effect was observed for OXA. Interestingly, PQQ4R at concentrations of 80 and $160 \mu \mathrm{M}$, significantly increased the antibacterial activity of OFX and TET in the AcrAB-overexpressing strain $A G 100_{\text {tet, }}$ (AcrAB overexpressed 6 to 10 times compared with the isogenic wild-type strain - Viveiros et al., 2005; Viveiros et al., 2007) causing a 4-fold decrease in their MICs, with a marginal effect on the MIC of EtBr. In accordance with the increased susceptibility detected above, the pump deficient AG100A did not grow in presence of PQQ4R at concentrations above $40 \mu \mathrm{M}$ and this concentration had no effect on the MIC of the antibiotics. However, this concentration caused a $\approx 8$-fold reduction in the EtBr MIC for AG100A, confirming that other efflux pumps, that extrude EtBr (AcrEF, among others), are active in this strain (Viveiros et al., 2005; Viveiros et al., 2007). 
314 The presence of PABN significantly reduced the MICs of OXA and TET on the three strains. PABN

315 reduced the MIC of TET and OXA on the pump-deficient strain by 16-fold and 4-fold,

316 respectively; however, it showed no effect on OFX and $\mathrm{EtBr}$ MICs. Conversely, the exposure to

$317 \mathrm{PABN}$ produced a 128-fold decreased in the MIC of EtBr against the AcrAB-overexpressing

318 strain, while no change was observed in the MIC of the wild-type strain. The inability of PABN to

319 reduce the MIC of EtBr in wild-type strains has already been described for $E$. coli and

320 Pseudomonas aeruginosa (Lomovskaya et al., 2001; Kern et al., 2006). This result indicates that

$321 \mathrm{PA} \beta \mathrm{N}$ is acting as a pump competitor and not as an efflux inhibitor like PQQ4R and CPZ. The

322 activity of $C P Z$ against the pump-deficient strain was similar to that of PQQ4R, with the

323 exception that CPZ reduced the MIC of TET by 4-fold. CPZ significantly reduced the MICS of OFX,

324 OXA, TET and EtBr on the wild-type and the AcrAB-overexpressing strain.

325

326 Efflux inhibitory activity of PQQ4R

327 The MIC results give an indirect measure of the potential efflux activity of the strains and the

328 effect of the inhibitors on this activity. The ability of PQQ4R to inhibit E. coli efflux systems was confirmed by real-time fluorometry using $\mathrm{EtBr}$, a broad efflux pump substrate (Viveiros et al., 2008; Viveiros et al., 2010). Initially, it was determined the equilibrium concentration at which the influx of EtBr equals its efflux. The accumulation of EtBr started at concentrations above 1 $\mu \mathrm{g} / \mathrm{ml}$ for $\mathrm{AG} 100,0.25 \mu \mathrm{g} / \mathrm{ml}$ for $\mathrm{AG} 100 \mathrm{~A}$, and $2 \mu \mathrm{g} / \mathrm{ml}$ for $\mathrm{AG}_{100}$ tet (Fig. S1). Using these concentrations, we evaluated the ability of PQQ4R, at sub-inhibitory concentrations (<1/8 MIC), to promote the intracellular accumulation of EtBr (Fig. 1) and calculated the RFFs (Table 3). The RFF value is a measure of how effective the compound is on the inhibition of the EtBr efflux (at a given concentration) by comparison of the final fluorescence at the last time point (60 minutes) of the treated cells with the cells treated only with EtBr. An index of activity above zero indicated that the cells accumulate more $\mathrm{EtBr}$ under the condition used than those of the control (non-treated cells taken as 0 ). In case of negative RFF values, these indicated that the treated cells accumulated less EtBr than those of the control condition. Values above 1 in the presence of the efflux inhibitors were considered enhanced accumulation of EtBr inside the 
342 cells. CPZ and PABN were included at the same concentrations for a direct comparison of their 343 inhibitory potencies.

344 At $80 \mu \mathrm{M}, \mathrm{PQQ} 4 \mathrm{R}$ showed an RFF of 13.1 against the wild-type strain, indicating a strong ability 345 to interfere with EtBr efflux compared to CPZ and PAßN, that showed RFF values of 8.1 and 0.7 , 346 respectively (Fig. 1A; Table 3). This means that PQQ4R promotes the higher accumulation levels 347 of EtBr compared to CPZ followed by PA $3 N$. In the AcrAB-deficient strain there was a small 348 increase in the accumulation of $\mathrm{EtBr}$ in the presence of PQQ4R (RFF=1.5) and CPZ (RFF=2), 349 whereas almost no effect was observed with PABN (RFF=0.1) (Fig. 1B; Table 3). These results 350 showed once again that $A c r A B$ is the main efflux system that pumps out $E t B r$ in $E$. coli, but that 351 other efflux pumps susceptible to PQQ4R and CPZ are pumping out EtBr as described before 352 (Viveiros et al., 2005; Viveiros et al., 2007). For the AcrAB-overexpressing strain AG100 ${ }_{\text {tet }}$ (AcrAB 353 is 6 to 10 times overexpressed compared to the wild-type strain), the accumulation of EtBr in 354 the presence of PQQ4R corresponded to a RFF of 5.2 (Fig. 1C; Table 3). The EtBr accumulation 355 by this strain increased significantly when compared to AG100A, but, for the same molar 356 concentration of inhibitor tested $(80 \mu \mathrm{M})$, did not reach the same levels of inhibition observed 357 for $A G 100$, since the $A c r A B$ system is 6 to 10 times more expressed in the $A G 100_{\text {tet }}$ strain compared with the AG100 (Viveiros et al., 2008; Viveiros et al., 2010). These results, obtained in a set of three isogenic $E$. coli that differ only by the absence/presence or expression level of the AcrAB system, indicated that PQQ4R promotes accumulation (i.e., interferes with the efflux) of $\mathrm{EtBr}$ in $E$. coli mainly thought the inhibition of the AcrAB efflux system. In the presence of $\mathrm{CPZ}$ and $P A B N$, the EtBr accumulation in the $A G 100_{\text {tet }}$ reaches similar levels (RFF 7.9 and 1.3, respectively) to those obtained for the wild-type strain (RFF 8.1 and 0.7 , respectively), being less effective in the inhibition of efflux activity than PQQ4R.

To confirm the results obtained above on the accumulation of EtBr promoted by the inhibitors, we performed efflux assays for each strain. Each strain was subject to conditions that promote significant EtBr accumulation over a period of 60 minutes at room temperature: in the absence of glucose and presence of PQQ4R, CPZ or PA $\beta N$. After the maximum accumulation has been reached, EtBr and the efflux inhibitors were washed-out and the cells were subsequently resuspended in new buffer with and without glucose and the inhibitor. As showed in Fig. 2, the 
371 efflux take place readily in presence of glucose at $37^{\circ} \mathrm{C}$, an activity that is inhibited in the

372 presence of PQQ4R and CPZ and to a lesser extent with PA $\beta N$. These results confirmed that

$373 \mathrm{PQQ} 4 \mathrm{R}, \mathrm{CPZ}$, and PAßN inhibit the efflux of EtBr and this inhibitory effect is transient when the

374 cells are washed-out of the inhibitor and an energy source is given to promote active efflux

375 reenergizing the cells (Fig. 2).

376

377 Membrane depolarization and cell viability

378 To test the hypothesis that the interference with the bacterial energy metabolism is the cause

379 of the efflux inhibition by PQQ4R, the effect of this compound on the bacterial membrane

380 potential was evaluated using the BacLight Bacterial Membrane Potential Kit. Thirteen minutes

381 after adding PQQ4R at any of the concentrations tested, the percentage of depolarized cells

382 was over $85 \%$ (Fig. 3A). The protonophore CCCP was used as depolarization control and, as

383 expected, the membrane potential collapsed when the cells were incubated for the same time

384 with CCCP (over 95\%). These results showed that PQQ4R depolarizes E. coli membranes by

385 interfering with the gradient of protons through the cell membrane. Then, to evaluate if the

386 depolarization of the cells, promoted by PQQ4R, results on a transient effect on cells with an

387 intact membrane or alters the membrane integrity, the Live/Dead BacLight Bacterial Viability Kit

388 was used. This assay uses the fluorescent stain SYTO 9 that penetrates in all bacterial

389 membranes and stains the cells in green, and propidium iodide that penetrates only in the cells

390 with permeabilized membranes. The combination of the two stains produces red fluorescent cells and these latter ones are considered dead by cell damage. The integrity of $E$. coli membranes was evaluated, by fluorometry, after exposure to PQQ4R at sub-inhibitory (1/4 and $1 / 2 \mathrm{MIC}$ ) and bactericidal concentrations (MIC), during 1 hour. The results showed that at half MIC, PQQ4R caused cell damage, reducing the membrane integrity by $28 \%$ (Fig. 3B). This increased membrane permeability was accompanied by a reduction in the bacterial viability of approx. 1 log (Fig. 4 - see also kinetics of bacterial killing). Membrane integrity was then evaluated on the cells exposed to PQQ4R at $80 \mu \mathrm{M}(\approx 1 / 8 \mathrm{MIC})$, the same concentration used in the real-time fluorometry accumulation and efflux assays, for 1 hour and the results were assessed as described before. At $80 \mu \mathrm{M}$, PQQ4R did not alter the cell membrane integrity (Fig. 
400

401

402

403

404

405

406

407

408

409

410

411

412

413

414

415

416

417

418

419

420

421

422

423

424

425

426

427

3B). To evaluate if the EtBr accumulation observed in presence of $C P Z$ and $P A B N$ was due to membrane permeability we tested both compounds also at $80 \mu \mathrm{M}$. The results showed that the membrane permeability increased by $18 \%$ and $14 \%$ with CPZ and PA $\beta N$, respectively. Membrane depolarization occurs in presence of $\mathrm{CPZ}$ at $80 \mu \mathrm{M}$ similarly to that caused by PQQ4R, and PAßN induced 14\% membrane depolarization at $80 \mu \mathrm{M}$. Overall, these results showed that PQQ4R causes transient membrane depolarization without affecting membrane integrity or cell viability at low concentrations $(80 \mu \mathrm{M}-1 / 8 \mathrm{MIC}$ - cell viability at this concentration $14.34 \log _{10} \mathrm{CFU} / \mathrm{ml}$ - Fig.4). In contrast, and as expected by the previous results, at bactericidal concentrations, PQQ4R acts as a membrane-destabilizing agent, which is also consistent with the cell death at this concentration (Fig. 4). The gathering of these results points towards the depolarization of the cell membrane as the main mechanism for PQQ4R's transient efflux-inhibition activity.

\section{PQQ4R effect on intracellular ATP levels}

Destabilization of the membrane functions can impair the respiratory chain functions and consequently reduce the ATP levels. To evaluate whether the exposure to PQQ4R could have an effect on the intracellular ATP levels, E. coli AG100 was exposed to PQQ4R and the intracellular ATP levels measured during 24 hours at either sub-inhibitory ( $320 \mu \mathrm{M}-1 / 2 \mathrm{MIC}$ ) or bactericidal concentrations (640 $\mu \mathrm{M}$ - MIC) (Fig. 5). The ATP levels remained constant during the first 3 hours of exposure at both concentrations and at the same level of the drug-free control excluding the abrupt ATP depletion from being the direct cause of the efflux-inhibition previously seen with the EtBr accumulation and efflux assays, and supports a slow but steady ATP production impairment. After 4 hours of exposure, the ATP levels of the drug-free control increased but the ATP levels of the drug-containing tubes remained practically constant after the addition of PQQ4R and until the end of the assay, with the exception of the cells exposed to half MIC. In this case, the ATP production increased after 24 hours of exposure but was $80 \%$ less than that of the drug-free control. 


\section{Kinetics of PQQ4R bacterial killing}

429 To characterize the killing effect of PQQ4R, we measured its bacterial killing activity against 430 AG100 through time-kill studies (Fig. 4). PQQ4R at half MIC had no effect on the viability of $E$. 431 coli after 24 hours of exposure. Exposure to PQQ4R reduced the viability of $E$. coli to zero after 4323 hours at the MIC $(256 \mu \mathrm{g} / \mathrm{ml}-640 \mu \mathrm{M})$ while at 2x MIC PQQ4R reduced the viability to zero 433 after 1 hour of exposure. After 24 hours of exposure, all cultures remained negative at these 434 concentrations. These results showed that PQQ4R killing activity is rapid reaching 100\% 435 lethality at the MIC, a concentration that was also found to be bactericidal (minimal bactericidal 436 concentration - MBC).

437

\section{Spectrum of activity of PQQ4R}

439 The antimicrobial activity of PQQ4R was evaluated against other bacterial species to assess its 440 antibacterial specificity: the Gram-negative bacteria Acinetobacter baumannii, Salmonella enterica serovar Enteritidis, Klebsiella pneumoniae and Enterobacter aerogenes, the Grampositive S. aureus, and the acid-fast bacteria Mycobacterium smegmatis, M. avium, and $M$. tuberculosis. The results are depicted in Table 4 and showed that PQQ4R possessed moderate antibacterial activity towards Gram-positive (average MIC $50 \mu \mathrm{g} / \mathrm{ml}$ ) and acid-fast bacteria (average MIC $32 \mu \mathrm{g} / \mathrm{ml}$ ) and reduced antibacterial activity against Gram-negative bacteria 446 (average $\mathrm{MIC} \geq 128 \mu \mathrm{g} / \mathrm{ml}$ ).

\section{Cytotoxicity}

Finally, PQQ4R was evaluated for its toxicity against human monocyte-derived macrophages using CPZ and PAßN for comparison. After 72 hours of exposure, the viability of the macrophages was assessed using the AlamarBlue method. The comparative activities, $\mathrm{CC}_{90}, \mathrm{CC}_{50}$ and $\mathrm{CC}_{10}$ values, are presented in Table 5. The dose-response curves are shown in Fig. 6. PQQ4R showed to be more toxic ( $\left.\mathrm{CC}_{50} 10.83 \mu \mathrm{M}\right)$ when compared to $\mathrm{CPZ}\left(\mathrm{CC}_{50} 22.24 \mu \mathrm{M}\right)$ and $\mathrm{PA} \beta \mathrm{N}$ $\left(C_{50} 1269 \mu \mathrm{M}\right)$. Afterwards, the amount of LDH release was measured in the cultures supernatants (Fig. 7). The LDH is an intracellular enzyme that is released in the culture media as consequence of damaged cell membranes (Chan et al., 2013). The treatment with PQQ4R at 
$4570.625 \mu \mathrm{M}$ to $2.5 \mu \mathrm{M}$ caused $1 \%$ to $4.6 \%$ increase in LDH release compared with the non-treated 458 cells; at $5 \mu \mathrm{M}$ LDH release increases by $52.45 \%$ and to $72 \%-78 \%$ at higher concentrations.

459 Comparatively, CPZ increases LDH release from $0.95 \%$ at $1.25 \mu \mathrm{M}$ to $24 \%$ at $20 \mu \mathrm{M}$. At

460 concentrations above, the amount of LDH detected in the culture supernatant was above $95 \%$.

461 Concerning PA $\beta N$, the amount of LDH detected varied from $1.08 \%$ to $8 \%$ at concentrations from

$46210 \mu \mathrm{M}$ to $640 \mu \mathrm{M}$. At concentrations above, PA $\mathrm{PN}$ caused $66 \%-68 \% \mathrm{LDH}$ release. The results

463 obtained with the LDH method corroborated the results obtained with the AlamarBlue method.

464

\section{DISCUSSION}

466 To detail the biological activity on the 2-phenylquinoline efflux inhibitor PQQ4R against E. coli, 467 we investigated first the synergistic activity of PQQ4R in combination with antibiotics that are 468 known substrates of the AcrAB efflux pump. The results showed that PQQ4R acts 469 synergistically, in a concentration dependent manner, with the antibiotics TET and OFX. When compared to $C P Z$ and $P A \beta N, P Q Q 4 R$ is less potent in its synergistic activity at equivalent molarities. Next, we explored the effect of PQQ4R on the real-time active efflux of the AcrAB efflux substrate EtBr. PQQ4R, CPZ, and PABN clearly inhibited the efflux of EtBr in a transient manner at sub-inhibitory concentrations (1/8 MIC or less), as shown by the reactivation of active efflux when the cells are released from the inhibitor and reenergized by glucose. As expected, the re-energization effect was more significant with CPZ (known to inhibit AcrABmediated EtBr efflux by energy depletion) (Viveiros et al., 2005) compared with PA $B N$, an AcrAB substrate competitor partially affecting membrane integrity (Misra et al., 2015). PQQ4R clearly demonstrated to have a real-time inhibitory effect on efflux similar to CPZ.

In terms of antimicrobial properties, PQQ4R demonstrated a rapid bactericidal activity against E. coli with the complete loss of viable cells after only 1 hour incubation at 2x MIC and after 4 hours at its MIC. This result is consistent with our hypothesis of an alternative mode of action in which at low concentrations PQQ4R inhibit E. coli efflux systems and near the MBC it interact directly with E. coli membranes. Our results showed that PQQ4R mechanism of action involves the loss of membrane integrity after 1 hour of exposure. PQQ4R disrupts both inner and outer 
486 membrane, as the uptake of the membrane-impermeant fluorescent dye propidium iodide 487 requires the damage of both to enter in the cytoplasm, to bind to the DNA and fluoresce.

488 Recently, it was showed that PAßN also permeabilizes the E. coli outer membrane (Misra et al., 4892015 ) and both outer and inner membranes of $P$. aeruginosa (Lamers, Cavallari \& Burrows, 490 2013).

491 The loss of the membrane integrity is closely related with the loss of the cell capacity to 492 synthesize ATP, decreased protein synthesis and to inhibit respiration (Brogden 2005). Our data 493 showed a moderate increase in the ATP levels after the addition of PQQ4R at sub-inhibitory 494 concentrations (half MIC), associated with a decrease in the membrane permeability. At 495 bactericidal concentrations (MIC), the ATP levels decreased and were accompanied by an 496 increase in the membrane permeability. To further support these results, and based upon the 497 fact that the RND efflux pumps act using the electrochemical gradient generated by the PMF 498 (Anes et al., 2015), we assessed whether treatment with PQQ4R could have a direct effect on membrane depolarization. Our results showed that PQQ4R causes a significant, but non-lethal, inner membrane depolarization at sub-inhibitory concentrations. At bactericidal concentrations, the membrane depolarization is accompanied by an impaired membrane integrity and ATP production, with cell death. The sequence of events describing PQQ4R mechanism of action is depicted in Fig. 8. Briefly, PQQ4R at low concentrations inhibit E. coli efflux systems by interfering with the energy necessary to maintain the pumps working, i.e, PQQ4R is an energetic inhibitor like CPZ and inhibits the efflux activity through the transient dissipation of the membrane potential without affecting the membrane permeability. Also similar to CPZ and PA $\beta N$, at higher concentrations PQQ4R permeabilizes $E$. coli membranes causing irreversible cell damage leading to the cell death. Of note, despite several attempts, we could not obtain E. coli mutants to PQQ4R. This result showed that PQQ4R has an off-target effect and corroborates our findings that points to membrane disruption as the primary mechanism of action of this molecule.

513 Regrettably, PQQ4R revealed to be relatively toxic to the human macrophage, with a toxicity 514 level comparable to that of $\mathrm{CPZ}$, an antipsychotic already used in clinical practice and previously 
515 shown to have activity against intracellular M. tuberculosis at non-toxic clinical concentrations

516 due to its accumulation inside the phagolysosomal macrophage compartments (Machado et al.,

517 2016). In a recent study, we showed that PQQ4R, when used at non-toxic concentrations, is

518 also able to potentiate clarithromycin activity against intracellular M. avium in a manner similar

519 to CPZ (Machado et al., 2015). On the contrary, PAßN showed almost no toxicity against the

520 human macrophages, however, PA $3 N$ and its derivatives proved to be nephrotoxic as result of

521 their accumulation in lysosomes. This adversity has hampered the clinical development of PAßN

522 (Watkins et al., 2003). The intriguing activity of PQQ4R may be used as a starting point for the

523 much-needed medicinal chemistry approaches to identify potent RND efflux pumps inhibitors

524 endowed of a suitable safety profile.

525

526 In conclusion, PQQ4R is an excellent hit candidate to be studied in depth to find new RND efflux

527 pumps inhibitors of Gram-negative bacteria that act by interfering with the PMF. It shows a

528 broad usefulness as efflux pump inhibitor and, due to its membrane permeabilizing properties,

529 can be used in combination therapies to assist other molecules to enter the bacterial cell

530 (Lamers, Cavallari \& Burrows, 2013; Herbel and Wink, 2016). Moreover, this compound

531 complies with the conditions of Farah's et al., (2013) hypothesis that presented an alternative

532 approach to tackle pathogens by using chemical combinations targeting the PMF. The authors

533 showed that combinations between dissipaters of membrane potential with dissipaters of the

534 transmembrane proton gradient are highly synergistic against methicillin-resistant S. aureus.

535 This combination will allow reducing each compound individual dose and consequently, their

536 toxicity (Farha et al., 2013). Since drug resistance mediated by efflux pumps depend largely on

537 the PMF, dissipaters of the PMF as PQQ4R could be regarded as putative adjuvants of the

538 conventional therapy against bacterial pathogens. Nevertheless, due to the similarities between

539 the bacterial and the mitochondrial electron transport chain, the effected of PQQ4R on the

540 latter will need to be evaluated in the future. Medicinal chemistry studies can now help to

541 improve PQQ4R scaffold to potentiate its permeabilizing and efflux inhibitory properties and

542 reduce its toxicity towards human cells, contributing for the development of new drugs with 
543 potential for the clinical usage as adjuvants of the therapy against drug resistant bacterial

544 pathogens.

545

546

\section{ACKNOWLEDGEMENTS}

548 DM, SSC, IC, and MV are thankful to Fundação para a Ciência e a Tecnologia (FCT), Portugal for 549 the support to the Global Health and Tropical Medicine (GHTM) Research Center.

550

\section{Supplemental Information}

552 Supplemental information for this article can be found online.

553 
554

555

556

557

558

559

560

561

562

563

564

565

566

567

568

569

570

571

572

573

574

575

576

577

578

579

580

581

582

\section{References}

Anes J, McCusker MP, Fanning S, Martins M. 2015. The ins and outs of RND efflux pumps in Escherichia coli. Frontiers in Microbiology 6:587. DOI 10.3389/fmicb.2015.00587.

Bohnert JA, Schuster S, Kern WV. 2013. Pimozine inhibits the AcrAB-TolC efflux pump in Escherichia coli. The Open Microbiology Journal 7:83-86. DOI 10.2174/1874285801307010083.

\section{Bohnert JA, Schuster S, Kern WV, Karcz T, Olejarz A, Kaczor A, Handzlik J, Kieć-Kononowicz.} 2016. Novel piperazine arylideimidazolones inhibit the AcrAB-TolC pump in Escherichia coli and simultaneously act as fluorescent membrane probes in a combined real-time influx and efflux assay. Antimicrobial Agents and Chemotherapy 60:1974-1983. DOI 10.1128/AAC.01995-15.

Brogden K. 2005. Antimicrobial peptides: pore formers or metabolic inhibitors in bacteria? Nature Reviews Microbiology 3:238-250. doi:10.1038/nrmicro1098.

Chan F, Moriwaki K, De Rosa MJ. 2013. Detection of necrosis by release of lactate dehydrogenase activity. Immune Homeostasis: Methods and Protocols. 979:65-70. DOI 10.1007/978-1-62703-290-2_7.

Clinical and Laboratory Standards Institute. 2014. Performance standards for antimicrobial susceptibility testing: twenty-fourth informational supplement, CLSI document M100-S24. CLSI, Wayne, PA; 2014.

\section{Coelho T, Machado D, Couto I, Maschmann R, Ramos D, von Groll A, Rossetti ML, Silva PA,} Viveiros M. 2015. Enhancement of antibiotic activity by efflux inhibitors against multidrug resistant Mycobacterium tuberculosis clinical isolates from Brazil. Frontiers in Microbiology 6:330. DOI 10.3389/fmicb.2015.00330. 
583

584

585

586

587

588

589

590

591

592

593

594

595

596

597

598

599

600

601

602

603

604

605

606

607

608

609

Du D, Wang Z, James NR, Voss JE, Klimont E, Ohene-Agyei T, Venter H, Chiu W, Luisi BF. 2014. Structure of the AcrAB-TolC multidrug efflux pump. Nature 509:512-515. DOI 10.1038/nature13205.

European Centre for Disease Prevention and Control. 2015. Annual epidemiological report 2014. Antimicrobial resistance and healthcare-associated infections. Stockholm, Sweden. ECDC, 2015.

Farha MA, Verschoor CP, Bowdish D, Brown ED. 2013. Collapsing the proton motive force to identify synergistic combinations against Staphylococcus aureus. Chemistry \& Biology 20:11681178. DOI 10.1016/j.chembiol.2013.07.006.

George AM, Levy SB. 1983. Amplifiable resistance to tetracycline, chloramphenicol, and other antibiotics in Escherichia coli: involvement of a non-plasmid-determined efflux of tetracycline. Journal of Bacteriology 155:531-540.

GraphPad Software QuickCalcs, 2016. Available online:

http://www.graphpad.com/quickcalcs/Ecanything1/ (accessed on 5 August 2016).

Herbel V, Wink M. 2016. Mode of action and membrane specificity of the antimicrobial peptide snakin-2. PeerJ 4:e1987. DOI 10.7717/peerj.1987.

Kern WV, Steinke P, Schumacher A, Schuster S, von Baum H, Bohnert JA. 2006. Effect of 1-(1naphthylmethyl)-piperazine, a novel putative efflux pump inhibitor, on antimicrobial drug susceptibility in clinical isolates of Escherichia coli. Journal of Antimicrobial Chemotherapy 57:339-343. DOI 10.1093/jac/dki445. 
610 Lamers R, Cavallari J, Burrows L. 2013. The efflux inhibitor Phenylalanine-Arginine Beta-

611 Naphthylamide (PABN) permeabilizes the outer membrane of Gram-negative bacteria. PLoS

612 One 8:e60666. DOI 10.1371/journal.pone.0060666.

613

614 Li XZ, Nikaido H. 2009. Efflux-mediated drug resistance in bacteria: an update. Drugs 69:1555-

615 1623. DOI 10.2165/11317030-000000000-00000.

616

617

Lomovskaya O, Warren M S, Lee A, Galazzo J, Fronko R, Lee M, Blais J, Cho D, Chamberlan S,

618 Renau T, Leger R, Hecker S, Watkins W, Hoshino K, Ishida H, Lee VJ. 2001. Identification and

619

characterization of inhibitors of multidrug resistance efflux pumps in Pseudomonas aeruginosa:

620

novel agents for combination therapy. Antimicrobial Agents and Chemotherapy 45:105-116.

621

DOI 10.1128/AAC.45.1.105-116.2001.

622

623

Machado D, Cannalire R, Costa SS, Manfroni G, Tabarrini O, Cecchetti V, Couto I, Viveiros M,

Sabatini S. 2015. The boosting effect of 2-phenylquinoline efflux inhibitors in combination with

macrolides against Mycobacterium smegmatis and Mycobacterium avium. ACS Infectious

626

Diseases 1:593-603. DOI 10.1021/acsinfecdis.5b00052.

627

628

Machado D, Pires D, Perdigão J, Couto I, Portugal I, Martins M, Amaral L, Viveiros M. 2016.

629

Ion channel blockers as antimicrobial agents, efflux inhibitors, and enhancers of macrophage

630 killing activity against drug resistant Mycobacterium tuberculosis. PloS one 11:e0149326. DOI

631 10.1371/journal.pone.0149326.

632

633

Machado L, Spengler G, Evaristo M, Handzlik J, Molnár J, Viveiros M, Kieć-Kononowicz K,

634

Amaral L. 2011. Biological activity of twenty-three hydantion derivatives on intrinsic efflux

635 pump system of Salmonella enterica serovar Enteritidis NCTC 13349. In Vivo 25:769-772.

636 
637 Misra R, Morrison KD, Cho HJ, Khuu T. 2015. Importance of real-time assays to distinguish 638 multidrug efflux pump inhibiting and outer membrane destabilizing activities in Escherichia coli. 639 Journal of Bacteriology 197:2479-2488. DOI 10.1128/JB.02456-14.

640

Nikaido H, Pagès JM. 2012. Broad-specificity efflux pumps and their role in multidrug resistance 642 of Gram-negative bacteria. FEMS Microbiology Reviews 36:340-363. DOI 10.1111/j.1574643 6976.2011.00290.x.

644

Nikaido H, Takatsuka Y. 2009. Mechanisms of RND multidrug efflux pumps. Biochimica et 646 Biophysica Acta (BBA)-Proteins and Proteomics 1794:769-781. DOI 10.1016/j.bbapap.2008.10.004.

648

Nishino K, Yamada J, Hirakawa H, Hirata T, Yamaguchi A. 2003. Roles of TolC-dependent 650 multidrug transporters of Escherichia coli in resistance to $\beta$-lactams. Antimicrobial Agents and Chemotherapy 47:3030-3033. DOI 10.1128/AAC.47.9.3030-3033.2003.

652

O'Brien J, Wilson I, Orton T, Pognan F. 2000. Investigation of the Alamar Blue (resazurin)

fluorescent dye for the assessment of mammalian cell cytotoxicity. European Journal of 655 Biochemistry 267:5421-5426. DOI 10.1046/j.1432-1327.2000.01606.x.

656

657

Okusu H, Ma D, Nikaido H. 1996. AcrAB efflux pump plays a major role in the antibiotic

resistance phenotype of Escherichia coli multiple-antibiotic-resistance (Mar) mutants. Journal of 659 Bacteriology 178:306-308.

660

661

Opperman TJ, Kwasny SM, Kim H-S, Nguyen ST, Houseweart C, D'Souza S, Walker GC, Peet 662 NP, Nikaido H, Bowlin TL. 2014. Characterization of a novel pyranopyridine inhibitor of the 663 AcrAB efflux pump of Escherichia coli. Antimicrobial Agents and Chemotherapy 58: 722-733. 664 DOI 10.1128/AAC.01866-13.

665 
666 Piddock ᄂ. 2006. Clinically relevant chromosomally encoded multidrug resistance efflux pumps

667 in bacteria. Clinical Microbiology Reviews 19:382-402. DOI 10.1128/CMR.19.2.382-402.2006.

668

669

Piddock L. 2007. Multidrug-resistance efflux pumps - not just for resistance. Nature Reviews

670

Microbiology 4:629-636. DOI 10.1038/nrmicro1464.

671

672

673

Pillai S, Moellering R, Eliopoulos G. 2005. Antimicrobial combinations. In: V. Lorian $5^{\text {th }}$ ed, Antibiotics in laboratory medicine, Lippincott Williams \& Wilkins, Philadelphia, PA, USA, 2005, $674 \quad 365-440$.

675

Pluta K, Morak-Młodawska B, Jeleń M. 2011. Recent progress in biological activities of synthesized phenothiazines. European Journal of Medicinal Chemistry 46:3179-3189. DOI 10.1016/j.ejmech.2011.05.013.

679

Sabatini S, Gosetto F, Manfroni G, Tabarrini O, Kaatz GW, Patel D, Cecchetti V. 2011. Evolution 681 from a natural flavones nucleus to obtain 2-(4-Propoxyphenyl)quinoline derivatives as potent inhibitors of the S. aureus NorA efflux pump. Journal of Medicinal Chemistry 54:5722-5736. DOI 10.1021/jm200370y.

684

Sabatini S, Gosetto F, Iraci N, Barreca ML, Massari S, Sancineto L, Manfroni G, Tabarrini O, 686 Dimovska M, Kaatz GW, Cecchetti V. 2013. Re-evolution of the 2-phenylquinolines: ligandbased design, synthesis, and biological evaluation of a potent new class of Staphylococcus aureus NorA efflux pump inhibitors to combat antimicrobial resistance. Journal of Medicinal Chemistry 56:4975-4989. DOI 10.1021/jm400262a.

690

Salih FA, Kaushik NK, Sharma P, Choudary GV, Murthy PS, Venkitasubramanian TA. 1991.

692

Calmodulin-like activity in mycobacteria. Indian Journal of Biochemistry and Biophysics 28: 491693 495. 
695 Schurig-Briccio LA, Yano T, Rubin H, Gennis RB. 2014. Characterization of the type 2 NADH:

696 menaquinone oxidoreductases from Staphylococcus aureus and the bactericidal action of

697 phenothiazines. Biochimica et Biophysica Acta (BBA)-Bioenergetics 1837:954-963. DOI

698 10.1016/j.bbabio.2014.03.017.

699

700

Thorsing M, Klitgaard JK, Atilano ML, Skov MN, Kolmos HJ, Filipe SR, Kallipolitis BH. 2013.

701

Thioridazine induces major changes in global gene expression and cell wall composition in

702

methicillin-resistant Staphylococcus aureus USA300. PloS One 8:e64518. DOI

703

10.1371/journal.pone.0064518.

704

705

Vargiu AV, Ruggerone P, Opperman TJ, Nguyen ST, Nikaido H. 2014. Molecular mechanism of 706 MBX2319 inhibition of Escherichia coli AcrB multidrug efflux pump and comparison with other

707 inhibitors. Antimicrobial Agents and Chemotherapy 58: 6224-6234. DOI 10.1128/AAC.03283-14.

708

709

Venter H, Mowla R, Ohene-Agyei T, Ma S. 2015. RND-type drug efflux pumps from Gram-

710 negative bacteria: molecular mechanism and inhibition. Frontiers in Microbiology 6:377. DOI

711 10.3389/fmicb.2015.00377.

712

713

Viveiros M, Jesus A, Brito M, Leandro C, Martins M, Ordway D, Molnar AM, Molnar J, Amaral

L. 2005. Inducement and reversal of tetracycline resistance in Escherichia coli K-12 and

715 expression of proton gradient-dependent multidrug efflux pump genes. Antimicrobial Agents

716 and Chemotherapy 49:3578-3582. DOI 10.1128/AAC.49.8.3578-3582.2005.

717

Viveiros M, Rodrigues L, Dupont M, Martins M, Couto I, Davin-Regli A, Martins M, Pagès JM, Amaral L. 2007. Antibiotic stress, genetic response and altered permeability of E. coli. PLoS One 2:e365. DOI 10.1371/journal.pone.0000365.

721 
724 automated ethidium bromide method. International Journal of Antimicrobial Agents 31:458-

725 462. DOI 10.1016/j.ijantimicag.2007.12.015.

726

727 Viveiros M, Rodrigues L, Martins M, Spengler G, Martins A, Amaral L. 2010. Evaluation of

728 efflux activity of bacteria by a semi-automated fluorometric system. In: Gillespie, S. H. and

729 McHugh, T. D., $2^{\text {nd }}$ ed, Antibiotic Resistance Protocols, Humana Press, Springer Science \&

730 Business Media, LLC, Secaucus, NJ, USA, 2010, 159-172. DOI 10.1007/978-1-60327-279-7_12.

731

732

Watkins WJ, Landaverry Y, Léger R, Litman R, Renau TE, Williams N, Rose Y, Zhang JZ,

733

Chamberland S, Madsen D, Griffith D, Tembe V, Huie K, Griffith D. 2003. The relationship

734

between physicochemical properties, in vitro activity and pharmacokinetic profiles of analogues

735

of diamine-containing efflux pump inhibitors. Bioorganic \& Medicinal Chemistry Letters

736

13:4241-4244. DOI 10.1016/j.bmcl.2003.07.030.

737

738

Weinstein E, Yano T, Li L, Avarbock D, Avarbock A, Helm D, McColm AA, Duncan K, Lonsdale

739

JT, Rubin H. 2005. Inhibitors of type II NADH: menaquinone oxidoreductase represent a class of antitubercular drugs. Proceedings of the National Academy of Sciences of the United States of America 122:4548-4553. DOI 10.1073/pnas.0500469102.

742

743

World Health Organization. 2014. Antimicrobial resistance global report on surveillance. 2014 summary. WHO/HSE/PED/AIP/2014.2. Genebra, Switzerland. World Health Organization, 2014.

Yilmaz S, Altinkanat-Gelmez G, Bolelli K, Guneser-Merdan D, Over-Hasdemir MU, Aki-Yalcin E,

Yalcin I. 2015. Binding site feature description of 2-substituted benzothiazoles as potential 
751 Zuo Z, Weng J, Wang W. 2016. Insights into the inhibitory mechanism of D13-9001 to the

752 multidrug transporter AcrB through molecular dynamics simulations. The Journal of Physical

753 Chemistry B 120:2145-2154. DOI: 10.1021/acs.jpcb.5b11942. 
Figure 1

Effect of PQQ4R, chlorpromazine, and PABN on the accumulation of ethidium bromide by $E$. coli

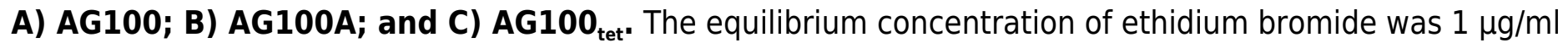
for $A G 100,0.25 \mu \mathrm{g} / \mathrm{ml}$ for $A G 100 A$, and $2 \mu \mathrm{g} / \mathrm{ml}$ for $A G 100_{\text {tet }}$. Assays were performed at $37^{\circ} \mathrm{C}$ in the absence of glucose. The inhibitors were used at $80 \mu \mathrm{M}$ for $\mathrm{AG} 100$ and $A \mathrm{G} 100_{\text {tet }}$ and $20 \mu \mathrm{M}$ for AG100A. EtBr, ethidium bromide; $C P Z$, chlorpromazine; $P A \beta N$, phenyl-arginine- $\beta$-naphthylamide.
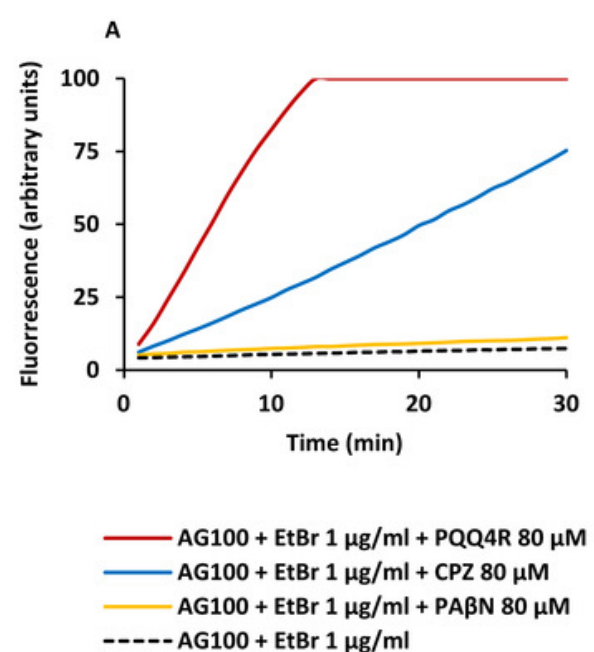
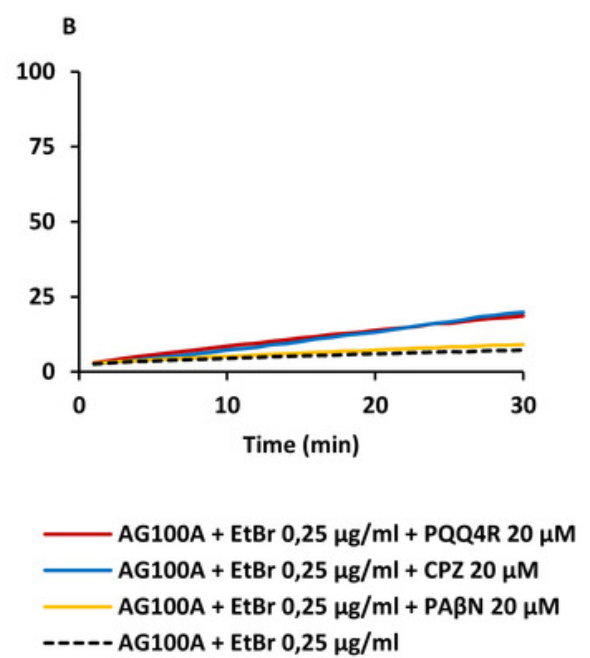
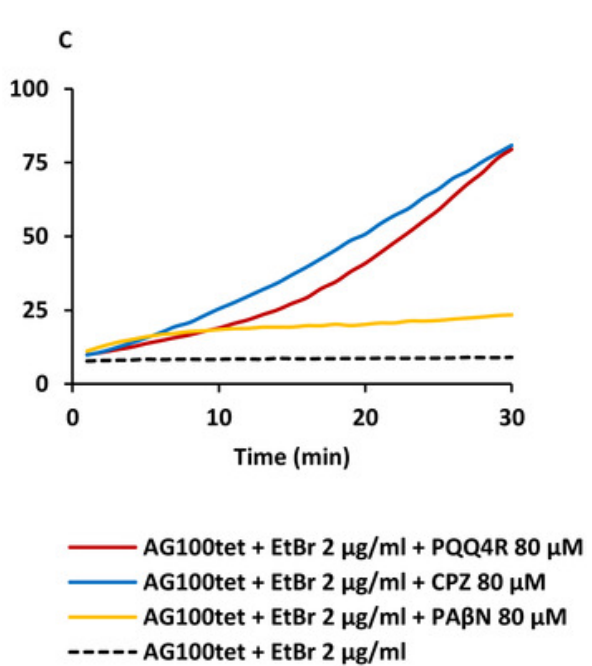
Figure 2

Effect of PQQ4R, CPZ, and PABN on the efflux of EtBr by the $E$. coli strains.

A) AG100; B) AG100A; and C) AG100 tet . The assays were performed at $37^{\circ} \mathrm{C}$ in the presence and absence of glucose. The concentrations of PQQ4R, CPZ and PABN were $80 \mu \mathrm{M}$ for $A G 100$ and $A{ }^{2} 100_{\text {tet }}$, and $20 \mu \mathrm{M}$ for AG100A. EtBr, ethidium bromide; CPZ, chlorpromazine; PAßN, phenyl-arginine- $\beta$-naphthylamide. 

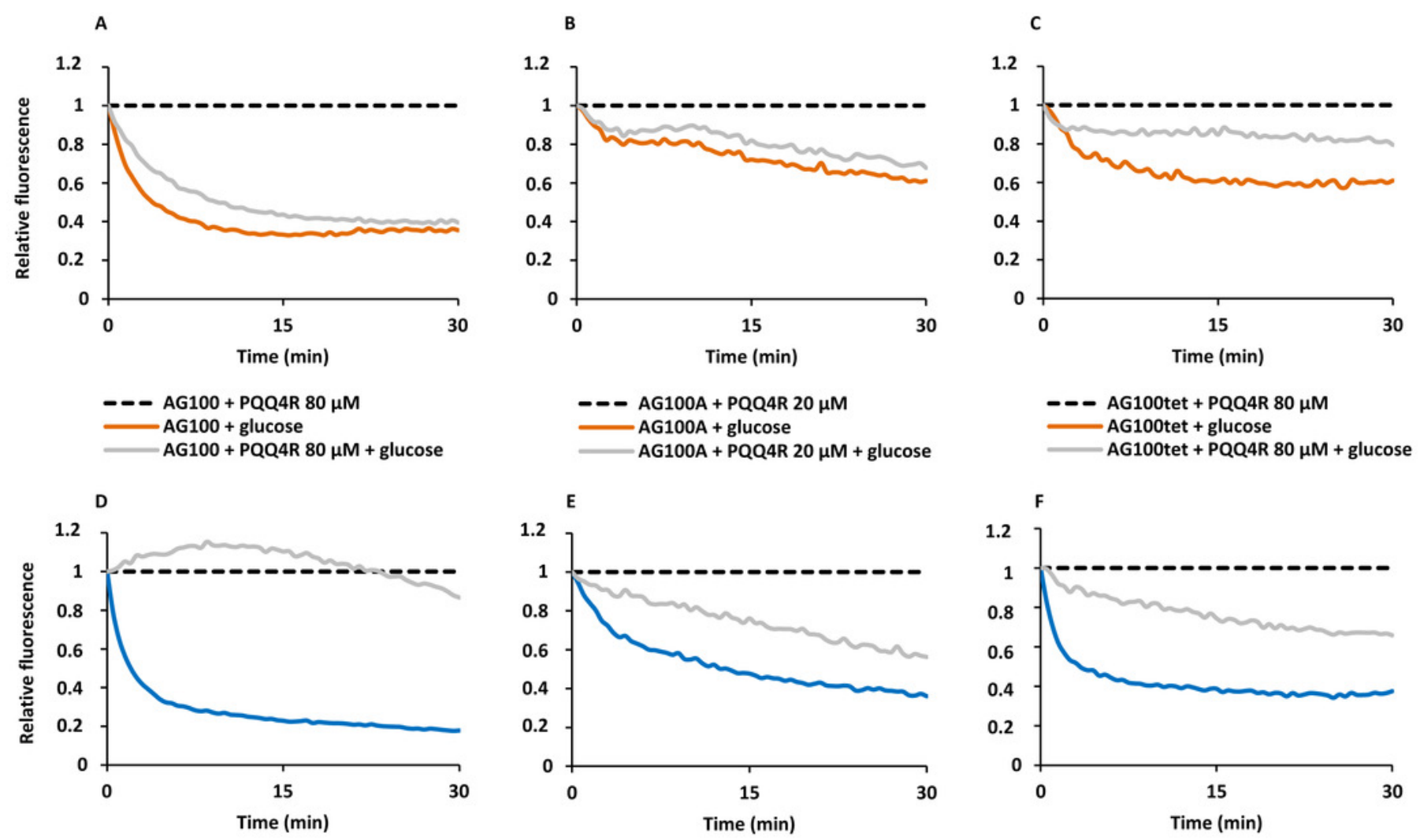

- - AG100 + CPZ $80 \mu \mathrm{M}$

AG100 + glucose

AG100 + CPZ $80 \mu \mathrm{M}+$ glucose

G

AG100A + glucose

- - AG100tet + CPZ $80 \mu \mathrm{M}$

AG100tet + glucose

AG100tet + CPZ $80 \mu \mathrm{M}+$ glucose

H
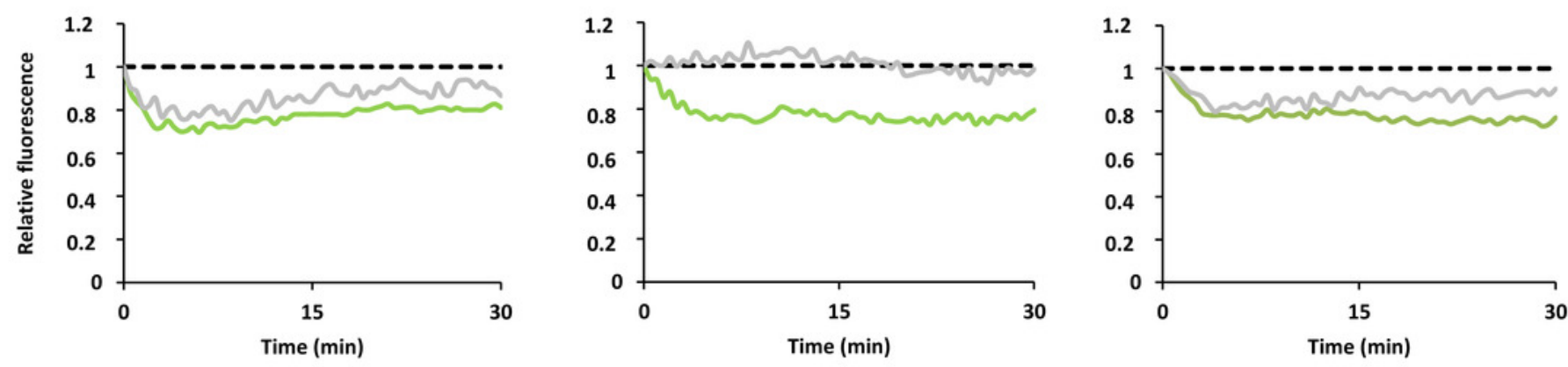

- - AG100+PABN $80 \mu \mathrm{M}$ AG100 + glucose

AG100 + PABN $80 \mu \mathrm{M}+$ glucose

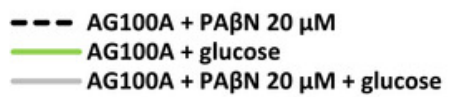

- AG100tet + PABN $80 \mu \mathrm{M}$
AG100tet + glucose
AG100tet + PABN $80 \mu \mathrm{M}+$ glucose 


\section{Figure 3}

\section{Membrane depolarization (A) and permeabilization (B).}

The relative red/green ratio of $E$. coli using $\mathrm{DIOC}_{2}(3)$ stained cells after 30 minutes of exposure to PQQ4R from $80 \mu \mathrm{M}$ to $640 \mu \mathrm{M}$. Green fluorescence corresponds to the depolarized cells; red fluorescence corresponds to the polarized cells. The changes in the fluorescence were measured at an excitation wavelength of 485/20 nm and 528/20 (emission) for green and 590/35 (emission) for red; B) Membrane permeability measured with Baclight Live/Dead assay upon exposure to PQQ4R. Green fluorescence corresponds to the cells with intact membranes; red fluorescence corresponds to cells with permeabilized membranes cells. The data was normalized against the drug-free control. CCCP was used as positive control, at $156 \mu \mathrm{M}$ (half MIC), since it eradicates the proton gradient, eliminating membrane potential. The results presented correspond to the average of two independent assays plus standard deviation ( \pm SD). Results were considered significant when $* \mathrm{P}<0.05$ and highly significant when $* * \mathrm{P}<0.01$ and $* * \mathrm{P}<0.001$.

A

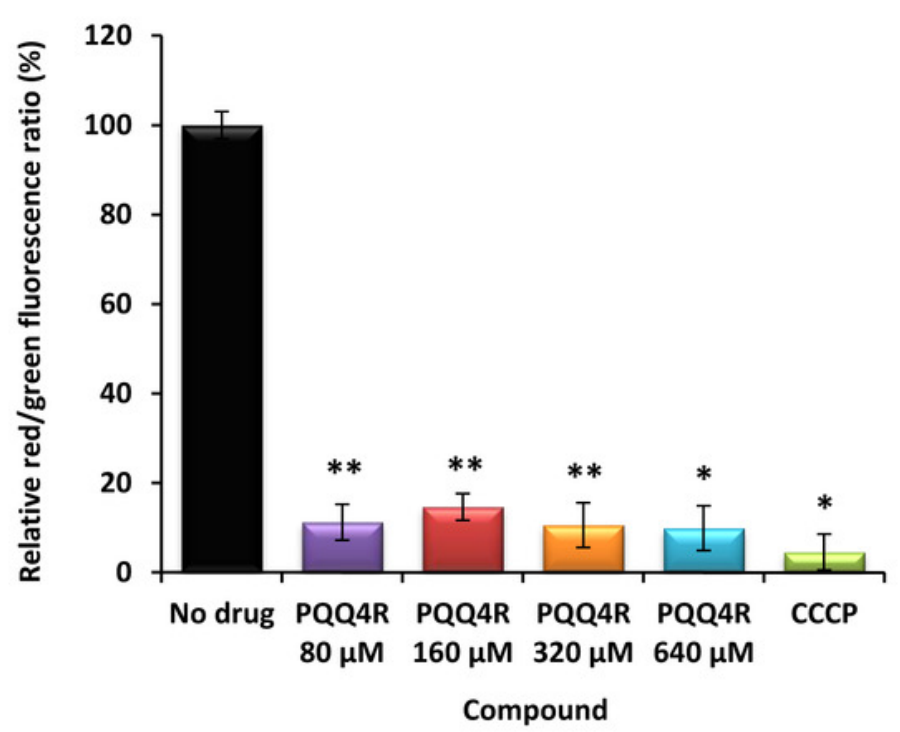

B

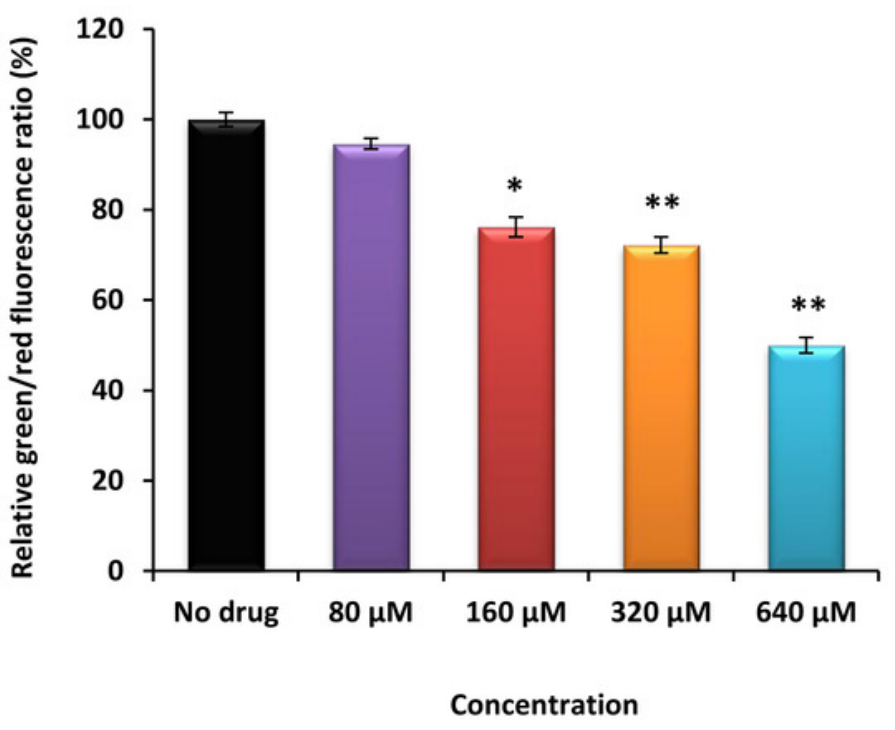


Figure 4

Effect for PQQ4R on survival of E. coli AG100.

The compound was added at concentrations from $0.5 \mathrm{x}$ MIC $(320 \mu \mathrm{M})$ to $8 \mathrm{x}$ MIC $(5120 \mu \mathrm{M})$ to exponential growing E. coli and survival monitored during 24 hours.

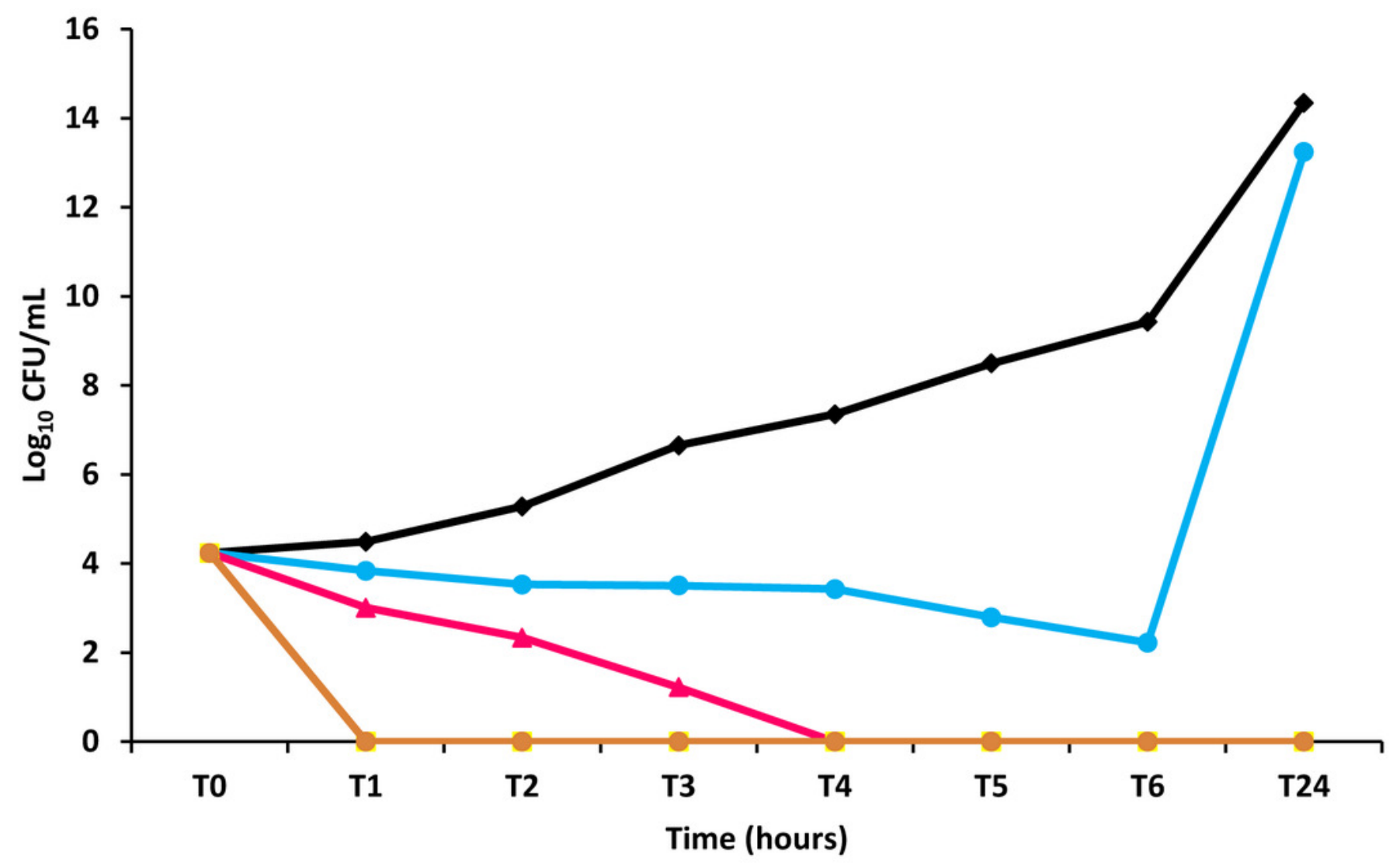

No drug

-PQQ4R half MIC

PQQ4R 4xMIC
-PQQ4R MIC

PQQ4R 8xMIC 


\section{Figure 5}

Effect of PQQ4R on E. coli ATP levels.

E. coli AG100 was exposed to PQQ4R at half MIC and MIC/MCB during 24 hours. The ATP levels were quantified using a luciferin-luciferase bioluminescence detection assay as described in Material and Methods. The results presented correspond to the average of two independent assays plus standard deviation $( \pm \mathrm{SD})$. Results were considered significant when $* \mathrm{P}<0.05$ and highly significant when $* * P<0.01$ and $* * * P<0.001$.

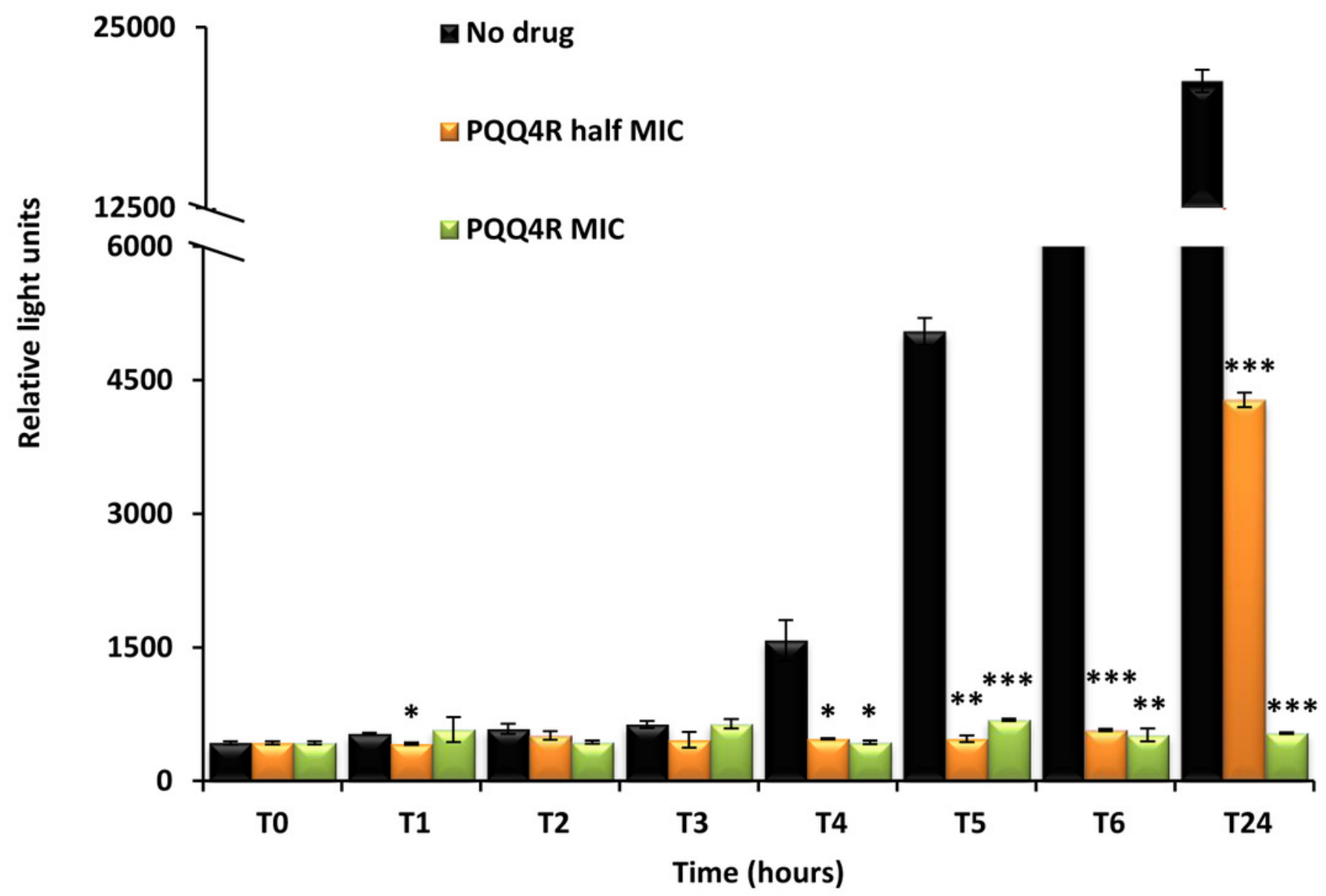


Figure 6

Dose response curves showing the effect of PQQ4R, CPZ and PABN against humanmonocyte derived macrophages.

The cells were treated with different concentrations of (A) PQQ4R, (B) CPZ, and (C) PAßN for 3 days; AlamarBlue was then added and cells were further incubated overnight at $37^{\circ} \mathrm{C}, 5 \% \mathrm{CO}_{2}$. The fluorescence was measured Synergy HT multi-mode microplate reader. CPZ, chlorpromazine; PAßN, phenyl-arginine- $\beta$ naphthylamide.
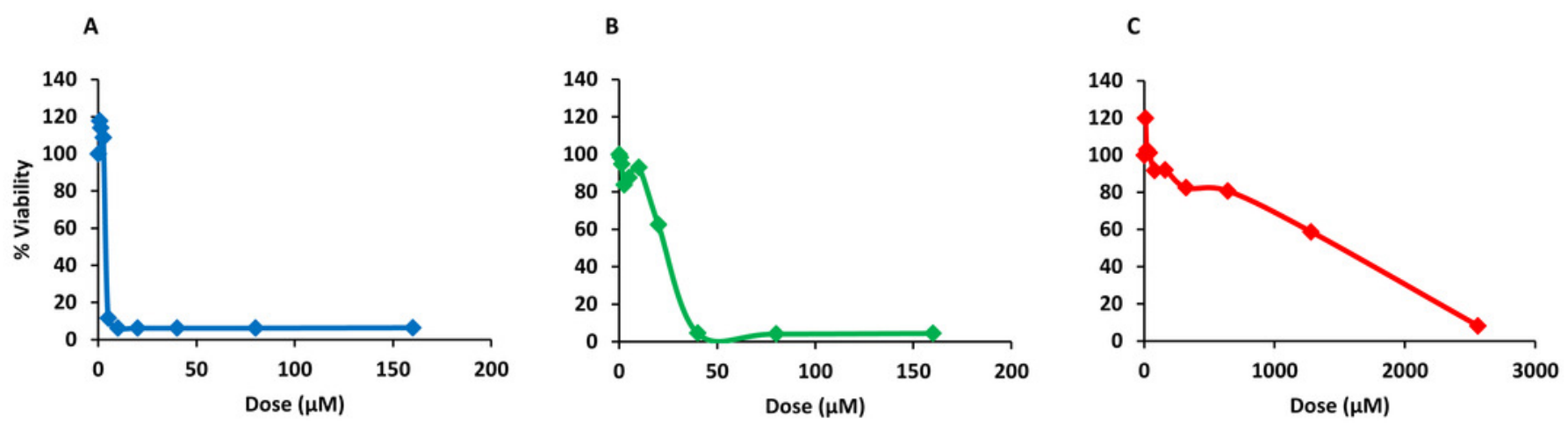
Figure 7

Effect of PQQ4R, CPZ and PABN on LDH release from human monocyte-derived macrophages.

The cells were exposed to each compound during 3 days at $37^{\circ} \mathrm{C}, 5 \% \mathrm{CO}_{2}$. The results presented correspond to the average of at least two independent assays plus standard deviation ( \pm SD). Results were considered significant when $* \mathrm{P}<0.05$ and highly significant when $* * P<0.01$ and $* * * P<0.001$.

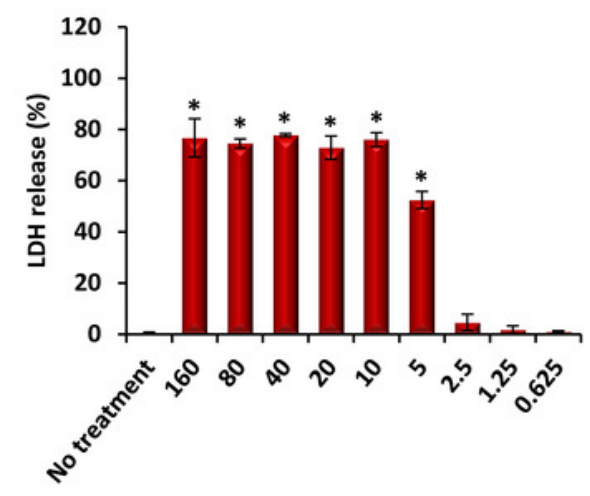

PQQ4R ( $\mu \mathrm{M})$

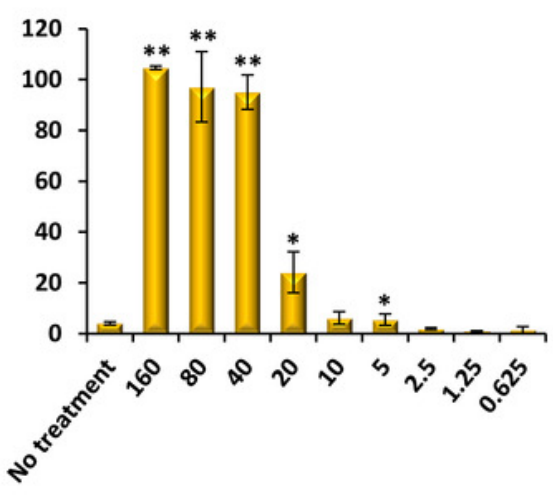

$\mathrm{CPZ}(\mu \mathrm{M})$

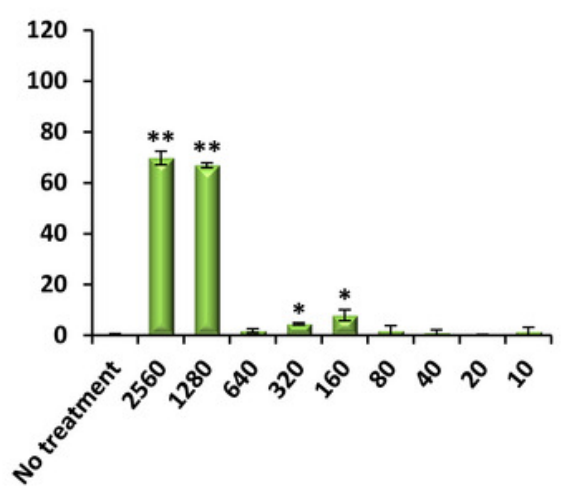

$\operatorname{PABN}(\mu \mathrm{M})$ 


\section{Figure 8}

Sequence of events describing PQQ4R mechanism of action.

PQQ4R mode of action involves efflux pump inhibition, membrane permeabilization, disruption of membrane potential, and ATP depletion. PQQ4R act as efflux inhibitor at low concentrations $(80 \mu \mathrm{M})$ by interference with energy required for efflux due to its interference with inner membrane potential. At sub-inhibitory concentrations (160 $\mu \mathrm{M}$ and $320 \mu \mathrm{M})$, PQQ4R destabilizes $E$. coli membrane functions in a non-permanent manner as suggested by the reduction of ATP levels, depolarization of membrane potential and increased membrane permeabilization; at the MBC $(640 \mu \mathrm{M})$, membrane permeabilization increases and ATP is lost.

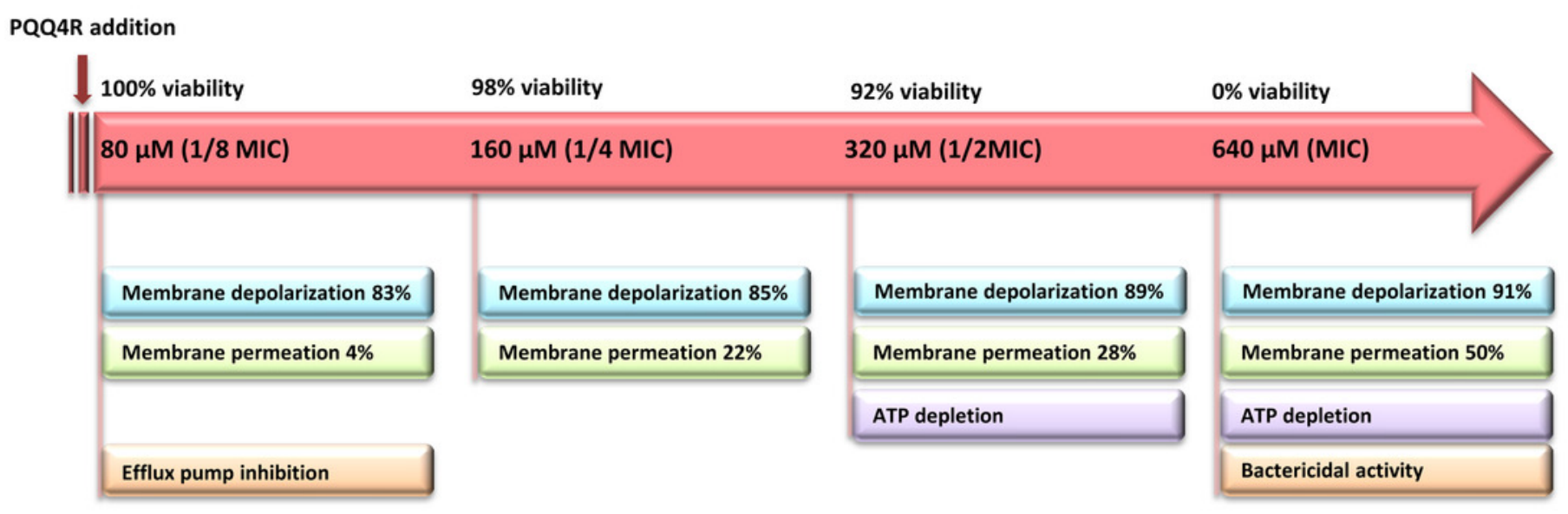




\section{Table $\mathbf{1}$ (on next page)}

Minimum inhibitory concentration (MIC) determination of the antibiotics, EtBr and efflux inhibitors against the $E$. coli strains. 
1 Table 1. Minimum inhibitory concentration (MIC) determination of the antibiotics, $\mathrm{EtBr}$ and 2 efflux inhibitors against the E. coli strains.

\begin{tabular}{|c|c|c|c|c|c|c|}
\hline \multirow{3}{*}{ Compound } & \multicolumn{6}{|c|}{ MIC for the $E$. coli strains } \\
\hline & \multicolumn{2}{|l|}{ AG100" } & \multicolumn{2}{|c|}{$\mathrm{AG} 100 \mathrm{~A}^{*}$} & \multicolumn{2}{|l|}{$A G 100_{\text {tet }^{2}}^{\&}$} \\
\hline & $(\mu \mathrm{g} / \mathrm{ml})$ & $(\mu \mathrm{M})$ & $(\mu \mathrm{g} / \mathrm{ml})$ & $(\mu \mathrm{M})$ & $(\mu \mathrm{g} / \mathrm{ml})$ & $(\mu \mathrm{M})$ \\
\hline \multicolumn{7}{|l|}{ Antibiotics } \\
\hline OFX & 0.25 & - & 0.0313 & - & 1 & - \\
\hline TET & 2 & - & 0.5 & - & 64 & - \\
\hline OXA & 512 & - & 2 & - & $>2048$ & - \\
\hline \multicolumn{7}{|c|}{ Efflux inhibitors } \\
\hline PQQ4R & 256 & 653.9 & 32 & 81.7 & $>256$ & $>653.9$ \\
\hline $\mathrm{CPZ}$ & 120 & 337.8 & 60 & 168.9 & 280 & 788 \\
\hline PABN & $>200$ & $>385$ & 50 & 96.3 & $>200$ & $>385$ \\
\hline \multicolumn{7}{|c|}{ Efflux substrate } \\
\hline EtBr & 200 & - & 3.125 & - & 512 & - \\
\hline
\end{tabular}

\section{Notes:}

\# E. coli AG100 - wild-type;

*E. coli AG100A - AG100 with the AcrAB-TolC efflux pump inactivated;

\& E. coli $A G 100_{\text {tet }}-A G 100$ with efflux pump overexpression;

$\mathrm{CPZ}$, chlorpromazine; $\mathrm{EtBr}$, ethidium bromide; OFX, ofloxacin; OXA, oxacillin; PA $\beta N$, phearg- $\beta$-naphthylamide; TET, tetracycline. 
Table 2 (on next page)

Synergistic effect of PQQ4R, PABN, and CPZ on the MIC values of antibiotics and EtBr against the $E$. coli strains. 
Table 2. Synergistic effect of PQQ4R, PABN, and CPZ on the MIC values of antibiotics and $\mathrm{EtBr}$ against the $E$. coli strains.

\begin{tabular}{|c|c|c|c|c|c|c|c|c|c|c|c|c|c|c|c|c|}
\hline \multirow{4}{*}{ Strain } & \multicolumn{16}{|c|}{ MIC ( $\mu \mathrm{g} / \mathrm{ml})$} \\
\hline & \multirow{2}{*}{\multicolumn{4}{|c|}{$\begin{array}{l}\text { OFX } \\
{[P Q Q 4 R](\mu M)}\end{array}$}} & \multirow{2}{*}{\multicolumn{4}{|c|}{$\begin{array}{l}\text { OXA } \\
\text { PQQ4R] }(\mu \mathrm{M}) \\
\end{array}$}} & \multirow{2}{*}{\multicolumn{4}{|c|}{$\begin{array}{l}\text { TET } \\
\text { [PQQ4R] }(\mu \mathrm{M})\end{array}$}} & \multirow{2}{*}{\multicolumn{4}{|c|}{$\begin{array}{l}\text { EtBr } \\
{[\text { PQQ4R] }(\mu \mathrm{M})}\end{array}$}} \\
\hline & & & & & & & & & & & & & & & & \\
\hline & 0 & 40 & 80 & 160 & 0 & 40 & 80 & 160 & 0 & 40 & 80 & 160 & 0 & 40 & 80 & 160 \\
\hline AG100 & 0.25 & 0.125 & 0.125 & 0.125 & 512 & 512 & 512 & 512 & 2 & 1 & 1 & 1 & 200 & 200 & 100 & 50 \\
\hline AG100A & 0.03 & 0.03 & - & - & 2 & 1 & - & - & 0.5 & 0.25 & - & - & 3.125 & 0.39 & - & - \\
\hline \multirow[t]{3}{*}{ AG100 } & 1 & 0.5 & 0.25 & 0.25 & $>2048$ & $>2048$ & 2048 & 2048 & 64 & 16 & 16 & 16 & 512 & 512 & 512 & 512 \\
\hline & \multicolumn{4}{|c|}{$[\mathrm{PA} \beta \mathrm{N}](\mu \mathrm{M})$} & \multicolumn{4}{|c|}{$[\mathrm{PA} \beta \mathrm{N}](\mu \mathrm{M})$} & \multicolumn{4}{|c|}{$[\mathrm{PA} \beta \mathrm{N}](\mu \mathrm{M})$} & \multicolumn{4}{|c|}{$[\mathrm{PA} \beta \mathrm{N}](\mu \mathrm{M})$} \\
\hline & 0 & 40 & 80 & 160 & 0 & 40 & 80 & 160 & 0 & 40 & 80 & 160 & 0 & 40 & 80 & 160 \\
\hline AG100 & 0.25 & 0.06 & 0.06 & 0.06 & 512 & 64 & 64 & 64 & 2 & 2 & 1 & 0.06 & 200 & 100 & 100 & 100 \\
\hline AG100A & 0.03 & 0.03 & - & - & 2 & 0.25 & - & - & 0.5 & 0.03 & - & - & 3.125 & 3.125 & - & - \\
\hline \multirow[t]{3}{*}{$A G 100_{\text {tet }}$} & 1 & 0.06 & 0.03 & 0.03 & $>2048$ & 256 & 256 & 64 & 64 & 8 & 4 & 2 & 512 & 256 & 128 & 4 \\
\hline & \multicolumn{4}{|c|}{$[\mathrm{CPZ}](\mu \mathrm{M})$} & \multicolumn{4}{|c|}{$[\mathrm{CPZ}](\mu \mathrm{M})$} & \multicolumn{4}{|c|}{$[\mathrm{CPZ}](\mu \mathrm{M})$} & \multicolumn{4}{|c|}{$[\mathrm{CPZ}](\mu \mathrm{M})$} \\
\hline & 0 & 40 & 80 & 160 & 0 & 40 & 80 & 160 & 0 & 40 & 80 & 160 & 0 & 40 & 80 & 160 \\
\hline AG100 & 0.25 & 0.125 & 0.125 & 0.003 & 512 & 512 & 512 & $\leq 1$ & 2 & 1 & 0.5 & $\leq 0.03$ & 200 & 100 & 25 & $\leq 0.39$ \\
\hline AG100A & 0.03 & 0.015 & - & - & 2 & 2 & - & - & 0.5 & 0.125 & - & - & 3.125 & 0.39 & - & - \\
\hline AG $100_{\text {tet }}$ & 1 & 0.5 & 0.5 & 0.25 & $>2048$ & $>2048$ & 2048 & 1024 & 64 & 32 & 32 & 16 & 512 & 256 & 128 & 128 \\
\hline
\end{tabular}

3 Notes:

\# E. coli AG100 - wild-type;

*E. coli AG100A - AG100 with the AcrAB-TolC efflux pump inactivated;

\&E. coli $A G 100_{\text {tet }}-A G 100$ with efflux pump overexpression; $C P Z$, chlorpromazine; EtBr, ethidium bromide; OFX, ofloxacin; OXA, oxacillin; PAßN, phe-arg- $\beta$-naphthylamide; TET, tetracycline.

-, AG100A does not grow at concentration above $40 \mu \mathrm{M}$ of PQQ4R.

9 


\section{Table 3(on next page)}

Relative final fluorescence values (RFF) based on the accumulation of $\mathrm{EtBr}$ for the $E$. coli strains in the presence of the efflux inhibitors 
Table 3. Relative final fluorescence values (RFF) based on the accumulation of EtBr for the $E$. coli strains in the presence of the efflux inhibitors

\begin{tabular}{|c|c|c|c|c|c|}
\hline \multicolumn{2}{|c|}{ AG100\# } & \multicolumn{2}{|c|}{ AG100A* } & \multicolumn{2}{|c|}{$\mathrm{AG} 100_{\text {tet }} \&$} \\
\hline $\begin{array}{l}\text { Compound } \\
(80 \mu \mathrm{M})\end{array}$ & RFF & $\begin{array}{l}\text { Compound } \\
\text { (20 } 20 \mathrm{M})\end{array}$ & RFF & $\begin{array}{l}\text { Compound } \\
(80 \mu \mathrm{M})\end{array}$ & RFF \\
\hline PQQ4R & $13.1 \pm 0.04 * * *$ & PQQ4R & $1.5 \pm 0.1 * *$ & PQQ4R & $5.2 \pm 0.9 * * *$ \\
\hline CPZ & $8.1 \pm 1.1 * * *$ & $\mathrm{CPZ}$ & $2.0 \pm 0.04^{* *}$ & $\mathrm{CPZ}$ & $7.9 \pm 0.4^{* * *}$ \\
\hline $\mathrm{PA} \beta \mathrm{N}$ & $0.7 \pm 1.2^{*}$ & PABN & $0.1 \pm 0.1$ & PABN & $1.3 \pm 0.3^{* *}$ \\
\hline
\end{tabular}

3 Notes:

$4 \quad \#$ E. coli AG100 - wild-type;

$5 *$ E. coli AG100A - AG100 with the AcrAB-TolC efflux pump inactivated;

6 \&. coli $\mathrm{AG} 100_{\text {tet }}-\mathrm{AG} 100$ with efflux pump overexpression;

$\mathrm{CPZ}$, chlorpromazine; EtBr, ethidium bromide; PABN, phe-arg- $\beta$-naphthylamide;

Accumulation of EtBr at $1 \mu \mathrm{g} / \mathrm{ml}$ (AG100), $0.25 \mu \mathrm{g} / \mathrm{ml}$ (AG100A) and $2 \mu \mathrm{g} / \mathrm{ml}$ (AG100 ${ }_{\text {tet }}$ ) in the absence of glucose;

Values in bold type (RFF $\geq 1$ ) indicated enhanced accumulation of EtBr in presence of an efflux inhibitor. The results presented

correspond to the average of three independent assays plus standard deviation $( \pm \mathrm{SD})$. Results were considered significant when $* P$

$<0.05$ and highly significant when ${ }^{* *} P<0.01$ and ${ }^{* * *} P<0.001$;

$12 \mathrm{CPZ}$, chlorpromazine; PA $\beta N$, phe-arg- $\beta$-naphthylamide, EtBr, ethidium bromide. 


\section{Table 4(on next page)}

Antibacterial specificity of PQQ4R against a panel of selected bacterial species. 
1 Table 4. Antibacterial specificity of PQQ4R against a panel of selected bacterial species.

\begin{tabular}{|c|c|c|}
\hline \multirow{2}{*}{ Species } & \multirow{2}{*}{ Strain } & \multirow{2}{*}{$\begin{array}{c}\mathrm{MIC}(\mu \mathrm{g} / \mathrm{ml}) \\
\text { PQQ4R }\end{array}$} \\
\hline & & \\
\hline Enterobacter aerogenes & ATCC13048 & $>256$ \\
\hline Salmonella enterica serovar Enteritidis & NCTC13349 & 256 \\
\hline Escherichia coli $\mathrm{K}-12$ & AG100 & 256 \\
\hline Acinetobacter baumannii & ATCC19606 & 128 \\
\hline Klebsiella pneumoniae & FF4891 & $>256$ \\
\hline Staphylococcus aureus & ATCC25923 & 50 \\
\hline Mycobacterium smegmatis $\mathrm{mc}^{2} 155$ & ATCC700084 $^{\top}$ & 32 \\
\hline Mycobacterium avium & 104 & 32 \\
\hline Mycobacterium tuberculosis H37Rv & ATCC27294 ${ }^{\top}$ & 32 \\
\hline
\end{tabular}

2 
Table 5(on next page)

Cytotoxic concentrations (CC) of PQQ4R, PABN, and CPZ against human monocytederived macrophages after three days of exposure. 
1 Table 5. Cytotoxic concentrations (CC) of PQQ4R, PABN, and CPZ against human monocyte-

2 derived macrophages after three days of exposure.

\begin{tabular}{cccc}
\hline & \multicolumn{3}{c}{ Cytotoxic concentrations $(\mu \mathrm{M})$} \\
\cline { 2 - 4 } Compound & CC90 & CC50 & CC10 \\
\hline PQQ4R & 12.73 & 10.83 & 9.21 \\
CPZ & 37.01 & 22.24 & 13.36 \\
PABN & 3596.86 & 1269 & 447.71 \\
\hline
\end{tabular}

3 Notes:

$4 \quad \mathrm{CPZ}$, chlorpromazine; PA $\beta \mathrm{N}$, phe-arg- $\beta$-naphthylamide.

5 